\title{
Gut microbiota and age shape susceptibility to clostridial enteritis in lorikeets under human care
}

David Minich $^{1 *}$ (minichdavid@gmail.com)

Christopher Madden ${ }^{1 *}$ (madden.80@osu.edu)

Mauricio A. Navarro ${ }^{2,3}$ (mnavarrob@ucdavis.edu)

Leo Glowacki ${ }^{4}$ (justgowacky@gmail.com)

Kristen French-Kim ${ }^{1}$ (french-kim.1@ osu.edu)

Willow Chan ${ }^{5}$ (chan.882@osu.edu)

Morgan V. Evans ${ }^{1,6}$ (morgvevans@gmail.com)

Kilmer Soares ${ }^{1,7}$ (kilmerzootec2010@ hotmail.com)

Ryan Mrofchak ${ }^{1}$ (mrofchak.5@osu.edu)

Rushil Madan'1 (madan.48@buckeyemail.osu.edu)

Gregory A. Ballash ${ }^{1}$ (ballash.4@osu.edu)

Krista LaPerle ${ }^{1,8}$ (la-perle.1@osu.edu)

Subhadeep Paul ${ }^{4}$ (paul.963@osu.edu)

Yael Vodovotz ${ }^{5}$ (vodovotz.1@osu.edu)

Francisco A. Uzal² (fauzal@ucdavis.edu)

Margaret Martinez ${ }^{1,9}$ (maggiemartinez0106@gmail.com)

Jennifer Hausmann ${ }^{10}$ (JHausmann@ denverzoo.org)

Randall E. Junge ${ }^{11}$ (randy.junge@ columbuszoo.org)

Vanessa L. Hale ${ }^{1}$ (hale.502@osu.edu)

${ }^{1}$ Ohio State University College of Veterinary Medicine, Columbus, OH, USA

${ }^{2}$ California Animal Health \& Food Safety Lab, University of California, Davis, San Bernardino, CA, USA

${ }^{3}$ Instituto de Patología Animal, Facultad de Ciencias Veterinarias, Universidad Austral de Chile, Chile

${ }^{4}$ Ohio State University College of Arts and Sciences, Columbus, OH, USA

${ }^{5}$ Ohio State University College of Food, Agricultural, and Environmental Sciences, Columbus, $\mathrm{OH}, \mathrm{USA}$

${ }^{6}$ Ohio State University College of Public Health, Columbus, OH, USA

${ }^{7}$ Department of Animal Science, College of Agricultural Sciences (CCA), Federal University of Paraiba (UFPB), Areia, PB, Brazil

${ }^{8}$ Ohio State University Comparative Pathology \& Digital Imaging Shared Resource, Columbus, $\mathrm{OH}, \mathrm{USA}$

${ }^{9}$ The Marine Mammal Center, Sausalito, CA, USA

${ }^{10}$ Denver Zoo, Denver, CO, USA

${ }^{11}$ Columbus Zoo and Aquarium, Columbus, $\mathrm{OH}$, USA

*contributed equally

\section{Corresponding author:}

Vanessa L. Hale, MAT, DVM, PhD

Veterinary Preventive Medicine Department

Ohio State University College of Veterinary Medicine 
bioRxiv preprint doi: https://doi.org/10.1101/2021.09.08.459483; this version posted September 8, 2021. The copyright holder for this preprint (which was not certified by peer review) is the author/funder, who has granted bioRxiv a license to display the preprint in perpetuity. It is made available under aCC-BY 4.0 International license.

\section{$47 \quad 1900$ Coffey Rd.}

48 Columbus, $\mathrm{OH} 43210$

49 hale.502@osu.edu

50 


\section{Gut microbiota and age shape susceptibility to clostridial enteritis in lorikeets under human care}

David Minich $^{1^{*}}$, Christopher Madden ${ }^{1 *}$, Mauricio A. Navarro ${ }^{2,3}$, Leo Glowacki ${ }^{4}$, Kristen French$\mathrm{Kim}^{1}$, Willow Chan ${ }^{5}$, Morgan V. Evans ${ }^{1,6}$, Kilmer Soares ${ }^{1,7}$, Ryan Mrofchak ${ }^{1}$, Rushil Madan ${ }^{1}$, Gregory A. Ballash ${ }^{1}$, Krista LaPerle ${ }^{1,8}$, Subhadeep Paul ${ }^{4}$, Yael Vodovotz ${ }^{5}$, Francisco A. Uzal ${ }^{2}$, Margaret Martinez ${ }^{1,9}$, Jennifer Hausmann ${ }^{10}$, Randall E. Junge ${ }^{11}$, Vanessa L. Hale ${ }^{1}$

${ }^{1}$ Ohio State University College of Veterinary Medicine, Columbus, OH, USA

${ }^{2}$ California Animal Health \& Food Safety Lab, University of California, Davis, San Bernardino, CA, USA

${ }^{3}$ Instituto de Patología Animal, Facultad de Ciencias Veterinarias, Universidad Austral de Chile, Chile

${ }^{4}$ Ohio State University College of Arts and Sciences, Columbus, OH, USA

${ }^{5}$ Ohio State University College of Food, Agricultural, and Environmental Sciences, Columbus, $\mathrm{OH}, \mathrm{USA}$

${ }^{6}$ Ohio State University College of Public Health, Columbus, OH, USA

${ }^{7}$ Department of Animal Science, College of Agricultural Sciences (CCA), Federal University of Paraiba (UFPB), Areia, PB, Brazil

${ }^{8}$ Ohio State University Comparative Pathology \& Digital Imaging Shared Resource, Columbus, $\mathrm{OH}$, USA

${ }^{9}$ The Marine Mammal Center, Sausalito, CA, USA

${ }^{10}$ Denver Zoo, Denver, CO, USA

${ }^{11}$ Columbus Zoo and Aquarium, Columbus, $\mathrm{OH}$, USA

*contributed equally

\section{Abstract}

\section{Background}

Enteritis is a common cause of morbidity and mortality in lorikeets that can be challenging to

diagnose and treat. In this study, we examine gut microbiota in two lorikeet flocks with enteritis

(Columbus Zoo and Aquarium - CZA; Denver Zoo - DZ). Since 2012, the CZA flock has

experienced repeated outbreaks of enteritis despite extensive diet, husbandry, and clinical

modifications. In 2018, both CZA and DZ observed a spike in enteritis. Recent research has

revealed that the gut microbiota can influence susceptibility to enteropathogens. We

hypothesized that a dysbiosis, or alteration in the gut microbial community, was making some 
87 lorikeets more susceptible to enteritis, and our goal was to characterize this dysbiosis and

88 determine the features that predicted susceptibility.

89 Results

90 We employed 16S rRNA sequencing to characterize the cloacal microbiota in lorikeets $($ CZA $\mathrm{n}=$

$9167, \mathrm{DZ} n=24)$ over time. We compared the microbiota of healthy lorikeets, to lorikeets with

92 enteritis, and lorikeets susceptible to enteritis, with "susceptible" being defined as healthy birds

93 that subsequently developed enteritis. Based on sequencing data, culture, and toxin gene

94 detection in intestinal contents, we identified Clostridium perfringens type A (CZA and DZ) and

95 C. colinum (CZA only) at increased relative abundances in birds with enteritis. Histopathology

96 and immunohistochemistry further identified the presence of gram-positive bacilli and $C$.

97 perfringens, respectively, in the necrotizing intestinal lesions. Finally, using Random Forests and

98 LASSO models, we identified several features (young age and the presence of Rhodococcus

99 fascians and Pseudomonas umsongensis) associated with susceptibility to clostridial enteritis.

100 Conclusions

101 We identified $C$. perfringens type A and C. colinum associated with lorikeet necrohemorrhagic

102 enteritis at CZA and DZ. Susceptibility testing of isolates lead to an updated clinical treatment

103 plan which ultimately resolved the outbreaks at both institutions. This work provides a

104 foundation for understanding gut microbiota features that are permissive to clostridial

105 colonization and host factors (e.g. age, prior infection) that shape responses to infection.

107 Keywords

108 Clostridium, enteritis, lorikeets, gut microbiota, trypsin

109 Background 
111 free-living lorikeets and lories, and outbreaks have been reported at multiple zoological

112 institutions [1-5]. In an informal survey of 12 North American zoos that house 10 or more

113 lorikeets, 11 of the 12 zoos reported a history of enteritis in their flocks, and $50 \%$ of the zoos

114 reported recurrent outbreaks of enteritis (Junge, Hausmann, unpublished data). In at least five of

115 these zoos, repeated cultures failed to identify an etiologic agent, and a combination of broad-

116 spectrum antimicrobials were employed as empiric therapy. This approach increases the risk of

117 promoting antimicrobial resistance [6]; moreover, in many cases, it also failed to resolve the

118 outbreak or prevent recurrences of enteritis.

The microbiome is a collection of bacteria, Archaea, viruses, and microbial eukaryotes

120 that live on or in hosts such as lorikeets. The microbiome is increasingly being realized as a

121 source for biomarkers that predict disease and clinical outcomes [7-9] and serve as targets for

122 therapeutics [10] (e.g. probiotics, prebiotics, phage therapy, dietary modification, microbiota

123 transplants). Over the past decade, studies on human and animal microbiomes have increased

124 exponentially [11], and we have learned that these microbial communities are critical to host

125 health. The gut microbiome, for example, plays an important role in nutrient acquisition and

126 metabolism [12], immune development [13], and pathogen defense [14, 15]. There are a growing

127 number of studies on avian [16-18] and wildlife microbiota [19-21], and these studies are

128 providing key insights into health and disease using minimally or non-invasive sampling

129 techniques [22-25]. Microbiome studies are also being used to inform conservation efforts in

130 wildlife species [26-28]. Recent research has further revealed that the gut microbiota influences

131 susceptibility to enteropathogens. For example, Clostridium, Campylobacter, and Salmonella

132 species, all of which are common agents in avian enteritis [1, 2, 5, 29-34], are adept at invading 
133 dysbiotic (or already disrupted) microbial communities but not at colonizing healthy microbial

134 communities [35-37].

In this study, we examined the microbiota over time in two lorikeet flocks (Columbus

136 Zoo and Aquarium [CZA], Denver Zoo [DZ]) that experienced one or more outbreaks of

137 enteritis. Between 2012 and 2018, the CZA lorikeet flock experienced repeated outbreaks of

138 enteritis despite extensive efforts to resolve these issues through nutrition, sanitation, medication,

139 and habitat and husbandry modifications [38]. In 2018, lorikeet morbidity and mortality events

140 spiked, and necropsy reports consistently identified severe and necrotizing enteritis in these

141 lorikeets, but bacterial cultures were frequently negative or variable (e.g. Escherichia coli,

142 Enterococcus spp.). Around the same time, DZ was also managing an outbreak of enteritis in

143 their lorikeet flock. We took a novel approach to lorikeet enteritis and sampled healthy and sick

144 birds at CZA and DZ over 10 months. We hypothesized that a dysbiosis, or alteration in the gut

145 microbial community, was making some lorikeets more susceptible to enteritis. Hence, our goal

146 was to characterize this dysbiosis and determine if and what features predicted susceptibility.

\section{Results}

\section{Columbus Zoo and Aquarium lorikeet enteritis}

151 We sampled a total of 67 lorikeets at the CZA between November 2018 and September 2019.

152 During this time, 34 lorikeets developed enteritis one or more times while the remaining birds (n

$153=33$ ) never developed enteritis (Table 1). Birds with enteritis were identified through clinical

154 signs (diarrhea, lethargic, fluffed). "Enteritis" samples collected from the same bird within two

155 weeks of the initial enteritis sample were considered a single case. Enteritis samples collected 
beyond 2 weeks from the initial sample in the same bird were counted as a second case of enteritis. There were no significant differences by sex in the number of lorikeets that did or did not develop enteritis $\left(\chi^{2}=5.7, p=0.06 ; \chi^{2}\right.$ between males and females only, excluding "unknown" $=0.12, p=0.73)$. However, there was a significant difference by age $\left(\chi^{2}=9.7, p=\right.$ $0.02)$ and by species $\left(\chi^{2}\right.$ with all species $=7.2, p=0.07 ; \chi^{2}$ between rainbow and coconut

161 lorikeets only (the two dominant species in this flock) $=6, p=0.01$ ), with enteritis occurring 162 more in younger birds (<2 years old) and in coconut lorikeets. Birds that survived a previous 163 episode of enteritis also had a 2.2 times increased risk of developing future enteritis; although,

164 this was only marginally significant [95\% CI: 0.99-5.29; $\mathrm{p}=0.051]$. We also observed an increase in enteritis cases between December 2018 and March 2019 (Fig. 1a).

\section{Gut microbiota by demographics and season}

168 We did not observe significant differences in microbial composition or diversity (Shannon,

169 Observed Features) by sex or species; although, by age, some differences in composition were 170 detected via the Unweighted, but not the Weighted UniFrac metric (Additional File 4, alpha-

171 diversity, all Kruskal-Wallis $p>0.05$; Additional File 5). There were also significant differences

172 in microbial composition and diversity by season (November 2018, February 2019, September

173 2019) with diversity declining significantly over time (Fig. 1b,c, Weighted UniFrac,

174 PERMANOVA $p=0.001$; Observed Features Kruskal-Wallis, all $p<0.001$, Additional File 6).

\section{Gut microbiota changes with enteritis}

177 As compared to healthy lorikeets, we observed a significant decrease in microbial diversity and 178 altered microbial composition in lorikeets with enteritis or lorikeets that died or were euthanized 
179 as a result of enteritis (denoted "post-mortem” lorikeets) (Fig. 2, Observed Features Kruskal-

180 Wallis all $p<0.0005$, Weighted UniFrac PERMANOVA $p=0.001$; Additional File 7). The top

181 differentially abundant microbe between healthy birds and birds with enteritis was Clostridium

182 perfringens, which was significantly increased in lorikeets with enteritis (Fig. 2c; ANCOM

$183 \mathrm{~W}=1098$, no post-mortem birds included in this analysis). There were three other differentially

184 abundant clostridia, including $C$. colinum, $C$. neonatale, and $C$. paraputrificum which were also

185 all increased in birds with enteritis (Additional File 8). We then performed this analysis on a

186 subset of birds $(\mathrm{n}=25)$ for which we had matched samples at both healthy and enteritis time

187 points and we again observed a significantly increased abundance of clostridia including $C$.

188 perfringens and $C$. neonatale in these birds when they had enteritis (Fig. 2c, ANCOM W $=788$,

189 Additional File 9).

191 Culture, genotyping, and susceptibility testing

192 After identifying clostridial DNA in our sequencing data, we employed anaerobic culture of

193 lorikeet intestinal contents to look for the presence of viable clostridia in the gut. Intestinal

194 content was collected from a total of 13 lorikeets that died or were euthanized due to enteritis.

195 Contents were cultured on TSA agar with 5\% sheep blood. All colonies with unique morphology

196 were picked between 24 - 48 hours and underwent MALDI-TOF identification. We identified

197 clostridia in 7 out of the 13 intestinal content samples including C. perfringens in 6 of these 7

198 samples (Table 2). Other microbes that were identified in culture included: Escherichia coli (in 3

199 out of 13 samples), and C. paraperfringens or C. baratii, C. paraputrificum, and C. disporicum

200 each in 1 out of 13 samples. We did not culture $C$. colinum in any sample. 
202 We then performed toxinotyping on the 6 C. perfringens isolates. All isolates contained the cpa

203 gene (encoding alpha toxin) and were identified as $C$. perfringens type A. Five out of 6 isolates

204 also contained the $c p b 2$ gene (encoding beta 2 toxin) (Table 2). Susceptibility testing was

205 performed on 2 isolates. Both isolates were susceptible to metronidazole and penicillin, and one

206 was also susceptible to clindamycin.

208 Pathology of necrotizing enteritis

209 We next evaluated the intestinal histopathology of lorikeets with enteritis to determine whether

210 we could identify $C$. perfringens in intestinal lesions. To do this, we examined formalin-fixed

211 paraffin-embedded (FFPE) blocks of lorikeet intestinal tissue from a total of 37 CZA lorikeets

212 that were submitted to The Ohio State University College of Veterinary Medicine between 2015

213 and 2019. Twenty-eight of these lorikeets had necrotizing enteritis while 7 died or were

214 euthanized due to unrelated causes (encephalitis-2, trauma-1, non-enteric mycobacteriosis-2, air

215 sacculitis-1, proventriculitis-1) (Table 3).

217 There were no significant differences by sex or species in the number of lorikeets that did or did

218 not have enteritis (by sex: Freeman-Halton extension of Fisher's Exact Test $p=0.73$; between

219 males and females only, excluding “unknown”, $p=0.06$; by species: Fisher's Exact Test $p=$

220 0.11). There was also no significant difference in average age between birds with and without

221 enteritis (Kruskal Wallis $p=0.13$ ); although, birds with enteritis were generally younger. We

222 also examined the number of enteritis deaths by month and observed an increase in cases during

223 summer (June-September) (Fig. 3a). 
225 The majority of lorikeets had a clinical history of sudden weight loss, diarrhea, or sudden death.

226 Most often, gross findings consisted of marked muscle wasting with a prominent keel (Fig. 3b),

227 and multiple severely dilated loops of intestines with thin walls and scant or watery contents

228 and/or gas (Fig. 3c). Other gross findings included tan opaque viscous coelomic fluid, intestinal

229 segments impacted with friable dark red contents, and thickened tan mesentery with multiple

230 small, white, firm to soft nodules within the intestines as well as on the serosa (Fig. 3d).

231

232 By histopathology, the most common finding (96\% of cases) was an intraluminal coagulum

233 comprised of red blood cells, bacterial colonies and sloughed necrotic mucosa (Fig 3e).

234 Approximately two thirds (64\%) of the cases had full thickness heterophilic and/or

235 granulomatous enteritis with ulceration (Fig 3f). Approximately two thirds of the cases (61\%)

236 had marked intestinal loop dilation, with over one third (39\%) having villous fusion and/or

237 blunting. Thirty six percent had fibrosis within the intestinal wall, most often at sites of

238 transmural inflammation and necrosis. The most commonly associated lesion was granulomatous

239 and/or heterophilic coelomitis $(86 \%)$ with half of those cases having intracoelomic bacteria,

240 most frequently bacilli or mixed bacteria. Other common lesions associated with necrotizing

241 enteritis included mild to moderate renal tubular necrosis and/or mineralization most likely due

242 to dehydration or septicemia (50\%) and marked extramedullary hematopoiesis within the liver

$243(61 \%)$. Evaluation of intestinal segments with modified Brown-Hopps Gram stain demonstrated

244 mixed bacteria within the necrohemorrhagic coagulum and/or necroulcerative intestinal lesions.

245 Eighty-nine percent of cases had large gram-positive bacilli present within the necrohemorrhagic

246 coagulum and these gram-positive bacilli were also the most abundant bacteria present, followed

247 by gram-positive cocci (71\%), and gram-negative bacilli (54\%) (Fig. 3g), whereas $57 \%$ of the 
transmural enteritis lesions contained large gram-positive bacilli, followed by $25 \%$ with grampositive cocci and $21 \%$ with gram-negative bacilli.

There was a strong correlation between the histopathologic characterization of lesions as chronic

252 versus acute with the clinical history of repeated versus first-time enteritis cases, respectively

253 (Fisher's exact test, $\mathrm{p}=0.033$; Relative risk 2.95, 95\% CI $=[0.86-10.08]$; Sensitivity $0.875,95 \%$

$254 \mathrm{CI}=[0.62-0.98])$. The chronicity of the lesions was assessed based on the presence of fibrosis

255 and mononuclear inflammatory infiltrates. There was no association between chronicity and the

256 presence of $C$. perfringens $\left(\chi^{2}=0.0009, p=0.98\right)$ or $C$. colinum $\left(\chi^{2}=0.36, p=0.55\right)$.

\section{Clostridium identification and toxinotyping in FFPE lorikeet intestinal samples}

259 We then used immunohistochemistry (IHC) and an anti-C. perfringens antibody to determine

260 whether the gram-positive bacilli present in intestinal lesions were $C$. perfringens. We identified of $7(14 \%)$ samples that had no enteritis (Table 4, Fig. 3h,i,j). PCR Toxinotyping of the FFPE intestinal tissue identified the C. perfringens alpha toxin gene (cpa) in 13 of the $28(46 \%)$ enteritis-positive samples and 1 of the 7 (14\%) samples with no enteritis lesions. No FFPE intestinal samples were positive for any of the following toxin genes: $c p b$ (beta), etx (epsilon), itx (iota), cpe (enterotoxin), or netB (necrotic B-like). Additionally, although the gene encoding cpb2 (beta-2 toxin) was identified in intestinal isolates, we did not find $c p b 2$ in the FFPE enteritis-positive intestines and $1(14 \%)$ of the samples with no lesions. 


\section{Denver Zoo lorikeet enteritis}

272 To determine whether $C$. perfringens could also be found in lorikeets with enteritis at other

273 institutions, we collected cloacal swabs and intestinal content from lorikeets at the DZ between

274 November 2018 and May 2019. We identified 12 birds that died or were euthanized due to

275 enteritis, and sampled these birds at necropsy ("post-mortem"). These birds were then age-, sex-,

276 and species-matched as closely as possible to 12 healthy lorikeets that were sampled during a

277 flock survey in May 2019 when all birds were reported to be healthy (Additional File 10).

278 Similar to the CZA lorikeets, we observed decreased microbial diversity and altered microbial

279 composition in the post-mortem lorikeets with enteritis as compared to healthy birds (Observed

280 Features Kruskal-Wallis $p<0.0005$, Weighted UniFrac PERMANOVA $p=0.001$, Fig. 4;

281 Additional File 11). C. perfringens was also significantly increased in relative abundance in the

282 post-mortem birds (Fig. 4c,d; ANCOM W =67). However, none of the DZ samples contained $C$.

283 colinum $16 \mathrm{~S}$ reads nor did they test positive for $C$. colinum via PCR. Type A C. perfringens was

284 also cultured from eight of the DZ birds with enteritis.

\section{Susceptibility to clostridial enteritis}

287 Odds and risk ratios associated with C. perfringens and C. colinum presence in healthy birds

288 After identifying clostridial enteritis in two separate lorikeet flocks, we then used the CZA

289 lorikeet data to explore factors that could be linked to susceptibility. First, we examined 16S

290 rRNA reads for the presence of $C$. perfringens and $C$. colinum in healthy birds to determine

291 whether they were predictors for developing future enteritis. Healthy CZA birds that had $C$.

292 perfringens $(\mathrm{n}=24,36 \%)$ or $C$. colinum $(\mathrm{n}=7,11 \%)$ present in their gut were not at increased

293 odds or risk of developing future enteritis (C. perfringens: $\mathrm{OR}=0.59,95 \% \mathrm{CI}=[0.21-1.62], \mathrm{p}=$ 
0.31; $\mathrm{RR}=0.76,95 \% \mathrm{CI}=[0.44-1.32], p=0.33 ;$ C. colinum: $\mathrm{OR}=1.37,95 \% \mathrm{CI}=[0.28-6.71]$, $\mathrm{p}=0.69 ; \mathrm{RR}=1.16,95 \% \mathrm{CI}=[0.58-2.32], p=0.67)$. Notably, $C$. perfringens and $C$. colinum were only present at low relative abundances $(<1 \%)$ in healthy birds, if present at all.

Gut microbial composition and diversity of susceptible birds

299 We then divided all healthy CZA birds into two groups: True Healthy and Susceptible. True

300 Healthy birds remained healthy throughout the entire sampling period (Nov. 2018 - Sept. 2019)

301 and never developed enteritis. Susceptible birds were healthy birds that went on to develop $C$.

302 perfringens enteritis at least during the sampling period. We compared the microbial communities of these groups and observed significantly increased microbial diversity and altered

304 microbial composition in Susceptible birds as compared to True Healthy birds or birds with enteritis (Observed Features Kruskal-Wallis $p<0.005$, Weighted UniFrac PERMANOVA $p=$ 0.001, Fig. 5; Additional File 12).

309 Next, we constructed Random Forests (RF) and LASSO models to compare True Healthy and

310 Susceptible birds based on samples collected during the February 2019 CZA flock survey (all

311 healthy birds). A total of 1503 microbial taxa (amplicon sequence variants - ASVs) were

312 included in these models along with demographic variables including lorikeet age and species.

313 Sex was not included as it was unknown for $31 \%$ of the birds. Seventy-five percent of the 314 samples were used as a training set and $25 \%$ of the samples were used as a test set. The RF 315 model identified the relative importance of variables as predictors (Additional File 12,c) while 316 the LASSO model identified whether a variable was associated with susceptibility or true health 
317 (Additional File 12,d). We then collated variables that were identified in both the RF and

318 LASSO models (Fig. 5c). The top 26 variables included 23 taxa associated with Susceptible

319 birds and 1 taxon (family Peptostreptococcaceae) associated with True Healthy birds. Rainbow

320 lorikeets (as opposed to coconut lorikeets) were also associated with health while the

321 "WasQuarantined TRUE" variable was associated with susceptibility. This variable represented

322 young lorikeets $(<1$ year old) that were transferred from another institution; these birds

323 underwent an initial quarantine prior to integration with the CZA flock. Some of the taxa

324 predictive of susceptibility included: Rhodococcus fascians, Kocuria spp., Pseudomonas

325 umsongensis, two taxa in the family Enterobacteriacea and an Aeromonas spp.

327 Dietary analysis for trypsin inhibitors

328 Finally, we examined lorikeet diets in relation to $C$. perfringens susceptibility. Several $C$.

329 perfringens toxins, including $c p a, c p b, p f o$, and $c p b 2$ (the toxin observed in 5 CZA $C$.

330 perfringens isolates) are sensitive to the host-produced protease trypsin [39]. However, trypsin

331 inhibitors present in the diet can block the activity of trypsin and thereby increase the risk of $C$.

332 perfringens toxin-mediated enteritis. As lorikeets are nectivores and their main diet under human

333 care consists of reconstituted powdered nectar, we opted to test trypsin inhibitor levels in six

334 commercial nectar brands including brands used at CZA and DZ. The range of trypsin inhibition

335 for the nectars was 0-1.79 trypsin inhibitor units (TIU)/mg dry nectar, denoting relatively low

336 inhibition (Fig. 6). For reference, raw soybeans, which have been linked to C. perfringens toxin-

337 mediated enteritis in poultry, contain approximately $46 \mathrm{TIU} / \mathrm{mg}$, and soy protein concentrate

338 contains $9.45 \mathrm{TIU} / \mathrm{mg}$ [40]. As such, the low levels of trypsin inhibition detected in commercial

339 nectars are unlikely to be playing a major role in susceptibility to $C$. perfringens enteritis in 
340 lorikeets; although we cannot rule out the possibility that other supplementary food items (e.g.

341 sweet potatoes or legumes) may have contributed to toxin-mediated clostridial enteritis. Notably,

342 C. colinum toxins have yet to be characterized; therefore, the role of trypsin and dietary trypsin

343 inhibitors on C. colinum pathogenesis is unknown.

\section{Discussion}

346 Our initial goal in this study was to characterize the gut microbiota of sick and healthy lorikeets

347 with the hypothesis that a dysbiosis was driving susceptibility to enteritis. While we did identify

348 gut microbial alterations associated with susceptibility, we also ended up identifying the

349 probable etiologic agents of enteritis in both the CZA and the DZ lorikeet flocks. Specifically,

350 we observed increased relative abundances of $C$. perfringens and C. colinum (CZA lorikeets

351 only) in the $16 \mathrm{~S}$ rRNA sequencing data. We then cultured lorikeet intestinal contents and

352 identified, genotyped, and susceptibility-tested multiple $C$. perfringens isolates. A

353 histopathologic examination of intestinal tissues further revealed inflammation, necrosis, and

354 ulcerative lesions that also contained gram-positive bacilli consistent with clostridial enteritis and

355 specifically C. colinum or C. perfringens. IHC and toxinotyping of intestinal tissues confirmed

356 the presence of $C$. perfringens in lorikeets with enteritis. PCR testing also confirmed the

357 presence of $C$. colinum in CZA lorikeets with enteritis. We then compared the gut microbiota of

358 healthy CZA lorikeets that developed enteritis to healthy CZA lorikeets that never developed

359 enteritis during our 10-month sampling period, and we identified several features associated with

360 susceptibility to enteritis including: age (younger birds are more susceptible), and increased

361 relative abundances of Rhodococcus fascians, Pseudomonas umsongensis, two taxa in the family

362 Enterobacteriacea, and an Aeromonas spp., among others. This work allowed us to identify the 
363 probable causative agents of lorikeet enteritis at two zoos, develop an optimal treatment plan

364 based on genotyping and susceptibility testing, and profile healthy birds at high risk of clostridial

365 enteritis.

366

367 Demographics of lorikeet enteritis

368 C. perfringens has been linked to enteritis in multiple mammal and bird species [34, 39, 41-44],

369 including in lorikeets and other psittacines [5, 32, 33, 45-47]. In this study, young lorikeets $(<2$

370 years old) were more likely to develop clostridial enteritis. We also observed some differences in

371 microbial composition by age (Additional File 5), and age emerged as a predictor of

372 susceptibility in the Random Forests model (Additional File 12,c). Previous reports in other

373 avian species note that the immunological naivete of young birds may increase susceptibility to

374 C. perfringens while adult birds are more resistant $[48,49]$. We also found that coconut lorikeets

375 were more likely to develop enteritis as compared to other lorikeet species. It is less clear what

376 may be driving species differences in clostridial enteritis; although, there were more coconut

377 lorikeets than any other species at CZA, and C. perfringens has been associated with necrotic

378 enteritis in coconut lorikeets at other institutions [47]. While type A was the dominant $C$.

379 perfringens toxinotype reported in the previous study on lorikeets, toxinotype C was also

380 common. Toxinotypes B, D, E, F, and G were also observed but less common [47]. Sex has also

381 been reported as a factor that influences susceptibility to necrotic enteritis in birds [41]; although,

382 we did not observe differences by sex in this study. Taken together, our results suggest that both

383 microbial and immunological factors may contribute to clostridial enteritis in young lorikeets.

\section{Seasonal changes in healthy lorikeet gut microbiota}


386 We observed flock-wide shifts in gut microbiota between the three flock surveys in November

387 2018, February 2019, and September 2019. In previous studies, seasonal alterations in avian

388 microbiota have been linked to diet, migration, and breeding status [21, 50-52]. Lorikeets in the

389 CZA flock have a consistent diet and environment year-round, and they do not migrate - thus

390 mitigating these factors as drivers for the observed seasonal changes. While breeding status

391 could be influencing these gut microbial alterations, it is also possible that flock-wide

392 prophylactic antimicrobial use during enteritis outbreaks and between flock surveys drove these

393 shifts. Notably, microbial diversity decreased significantly from November 2018 to September

3942019 , which is a shift that could be consistent with antimicrobial use across host species

395 including in birds [53-58].

\section{Lorikeet enteritis: microbes to host}

398 Lorikeets with enteritis demonstrated microbial community shifts and histopathological changes

399 as compared to healthy lorikeets. Based on 16S rRNA sequencing, culture, and genotyping, we

400 confirmed the presence and increased relative abundance of $C$. perfringens type A in CZA and

401 DZ lorikeets with enteritis. In the CZA lorikeets, we further determined that $C$. perfringens was

402 directly associated with necroulcerative intestinal enteritis via IHC and multiple intestinal

403 isolates of $C$. perfringens contained the $c p b 2$ toxin. C. colinum was also found at increased

404 relative abundances in CZA but not DZ birds based on 16S sequences and PCR. In both CZA

405 and DZ birds, we observed decreased microbial diversity and altered microbial composition in

406 lorikeets with enteritis as compared to healthy lorikeets. C. perfringens and C. colinum have

407 been implicated previously in necroulcerative enteritis in birds including lories, lorikeets, and

408 poultry [47, 59-63]. Microbial community alterations have also been reported in chickens 
infected with $C$. perfringens [64-67]. While several clostridial species are considered normal

410 flora in some avian species including poultry, in psittacines, clostridial species are rarely found

411 in the intestines of healthy birds, and taxa such as C. colinum and C. perfringens are considered

412 pathogenic $[4,31,59,68,69]$. Previous studies have linked the $C$. perfringens beta- 2 toxin

413 (cpb2) identified in CZA birds with enteritis in psittacines [33], storks [70], pigs [71], and

414 poultry [72]; however, the cpb2 toxin has also been identified in healthy individuals (poultry,

415 horses, dogs, and other avian species) and its role enteritis is not clear [73-77]. C. colinum-linked

416 enteritis has only been reported in avian species, but virulence factors, toxins, and disease

417 pathogenesis for C. colinum have yet to be fully elucidated [59].

419 Our results suggest that $C$. perfringens was the driver of enteritis in the DZ birds, while in the

420 CZA birds, either C. colinum, or C. perfringens, or both acting synergistically, could have been

421 driving the infections or creating a dysbiotic environment that allowed the other to thrive.

422 Synergistic C. perfringens / C. colinum co-infections have been reported previously in poultry

423 [78], while other clostridial co-infections (C. perfringens / C. difficile) have been reported in

424 humans, foals, and dogs [79-81]. Phylogenetically related bacterial taxa can share functional

425 traits and fill similar metabolic niches [82]; thus, it is feasible that an environment permissive to

426 one type of clostridia may also be permissive to another clostridia.

\section{Susceptibility to enteritis}

429 Besides age, seasonality has also been linked to necrotic enteritis in birds. In poultry, enteritis

430 cases occur more frequently in late winter and early spring [83-85], which is what we observed

431 in the CZA lorikeets; although historically, CZA reported enteritis cases across all seasons. DZ 
432 cases were also clustered during winter months, however this was the only occurrence of an

433 enteritis outbreak DZ had experienced. Additionally, birds that had a history of enteritis were at

4342.2 times increased risk of developing future enteritis. Gross pathology and histopathology data

435 further linked signs of chronic lesions (fibrosis, mucosal atrophy, villus blunting/fusion, wasting)

436 to birds with repeated cases of enteritis. Repeated bouts of enteritis in some CZA lorikeets likely

437 resulted in permanent intestinal damage that hindered absorption and peristalsis, leading to

438 malnutrition and intestinal stasis, which are known risk factors for C. perfringens enteritis [61,

$43986,87]$. While some lorikeets survived one or even 2 cases of enteritis, no bird lived beyond a

440 third episode.

442 Diet - and specifically dietary trypsin inhibitors - can also be a risk factor for C. perfringens

443 enteritis - depending on the toxinotype $[45,87,88]$. As such, we assessed trypsin inhibition in 6

444 commercial nectars including the nectars used at CZA and DZ. All nectars contained very low

445 levels of trypsin inhibitors and are unlikely to be contributing to enteritis incidence. However,

446 supplementary diet items, such as cooked sweet potatoes, which were briefly part of the DZ

447 lorikeet diet during the enteritis outbreaks, could potentially have contained higher levels of

448 trypsin inhibitors. Notably, trypsin inhibitor levels vary widely across sweet potato cultivars [89-

449 91], and we did not evaluate trypsin inhibition in any supplementary foods.

451 Finally, we examined microbial communities in healthy birds that later went on to develop

452 enteritis (Susceptible) or remained healthy throughout the entire study period (True Healthy).

453 Susceptible birds displayed minor but significant differences in microbial community diversity

454 (Observed Features), composition, and differentially abundant taxa. These microbial community 
455 differences could be linked to age as young birds were also the most susceptible. Although we

456 found no significant difference in microbial diversity or composition in healthy lorikeets by age,

457 birds in the youngest age group ( $<2$ years old) had the greatest microbial diversity (Additional

458 File 4, c,f) which was also true in the Susceptible birds (Fig. 5a). Increased microbial diversity

459 has also been observed in young chickens susceptible to $C$. perfringens infection as opposed to

460 those that were more resistant [92]. Moreover, differences in microbial composition by age

461 (Unweighted UniFrac, but not Weighted UniFrac) were significant in healthy birds $(p=0.01$

462 Additional File 5). This suggests that age may influence lorikeet microbial community structure,

463 and with a larger sample size, this may have been more apparent.

465 Whether shaped by age or not, the microbial community differences observed in Susceptible

466 birds suggest that a lorikeet's pre-existing microbial community structure could potentially

467 influence the ability of a clostridial pathogen (e.g. C. perfringens or C. colinum) to colonize the

468 intestinal tract. This could be achieved through alterations in the metabolic environment that

469 create a more favorable niche for clostridia to expand. The presence of primary bile acids, for

470 example, can act as a germinant for Clostridium species, while the presence of secondary bile

471 acids (produced by bacteria that convert primary to secondary bile acids), can inhibit $C$.

472 perfringens proliferation [93, 94]. Minor alterations in pre-existing microbial community

473 composition and differentially abundant microbes have also been reported in chickens

474 susceptible to $C$. perfringens [92].

475

476 We identified several microbes that were associated with susceptibility including Rhodococcus

477 fascians, Pseudomonas umsongensis, an Aeromonas spp., and two taxa in the family 
478 Enterobacteriacea. Rhodococcus fascians has been found at increased abundances in juvenile

479 birds (sparrows, < 1 year old) as compared to older birds and could be an age-related taxa [21]. A

480 single human case report also highlights a co-infection between $R$. fascians and a clostridial

481 species (C. difficile) [95], which leads to the intriguing question as to whether these 2 species

482 interact in ways that may support each other's growth. However, this co-infection was in an

483 immunocompromised individual, so the relevance is unclear. Both Pseudomonas and Aeromonas

484 species have been independently associated with enteritis in birds $[86,96,97]$. In a previous

485 study that employed a subcutaneous abscess model, the addition of Pseudomonas aeruginosa or

486 various Enterobacteriaceae species enhanced the growth of $C$. perfringens [98] suggesting that

487 interactions between these taxa may indeed facilitate clostridial infections. Our RF and LASSO

488 models also identified several other microbial taxa associated with susceptibility; although, the

489 potential role these taxa may be playing in clostridial infections or enteritis is undetermined and

490 requires additional study.

491

492 This study had several limitations: We identified both C. colinum and/or Type A C. perfringens

493 in lorikeets with enteritis; however, the mechanisms by which these bacteria caused disease

494 remain unclear. For example, while C. colinum has been empirically and experimentally linked

495 to ulcerative enteritis in birds, its virulence factors have yet to be elucidated [60]. Second,

496 although both $C$. perfringens alpha toxin (cpa) and beta-2 toxin (cpb2) have been associated with

497 enteritis in multiple host species including birds, the role of these toxins in enteritis pathogenesis

498 is ambiguous, and both of these toxin genes have been found in the intestines of healthy animals

$499[39,60,99]$. It is possible that neither $c p a$ nor $c p b 2$ are key virulence factors in Type A. $C$.

500 perfringens and that other unidentified virulence factors played a role in lorikeet enteritis. 
501 Additionally, toxin gene presence (e.g. PCR, used in this study) does not necessarily equate to

502 toxin gene expression. However, clostridia and its respective toxin genes are considered aberrant

503 in healthy psittacines $[4,31,59,68,69]$, suggesting that they are playing a role in enteritis even

504 if their virulence factors are not fully defined. The surrounding gut microbiota and metabolites

505 could also be mediating C. perfringens pathogenesis including colonization and toxin expression

506 as has been demonstrated in Clostridiodes difficile [35, 36]

507

508 Conclusions

509 In this study, we systematically examined gut microbiota and susceptibility to clostridial enteritis

510 in two lorikeet flocks under human care. A few of our key take-aways: 1) Clostridia can be

511 challenging to detect via culture in lorikeet cloacal swabs, but anaerobic culture of intestinal

512 contents yielded $C$. perfringens in 6 out of 13 isolates from CZA, and 16S sequencing allowed

513 ready identification of $C$. perfringens and $C$. colinum in birds with enteritis. As clostridia are not

514 normal inhabitants in psittacines, this was a significant finding. 2) Clostridial isolates then

515 underwent genotyping and susceptibility testing, which allowed us to update the lorikeets'

516 clinical treatment plans to more targeted therapies, aligned with antimicrobial stewardship

517 practices (DZ - metronidazole, CZA - florfenicol and clindamycin in clinically affected birds

518 and prophylactic flock-wide application of bacitracin). Since June of 2019, and as of this

519 writing, there have been no new cases of enteritis in lorikeets at either CZA or DZ. 3) Young age

520 (potentially linked with immunological naivete, limited exposures, or lower trypsin activity

521 [100]), prior enteritis, and specific microbes including $R$. fascians, $P$. umsongensis, and

522 Enterobacteriacea taxa are linked with susceptibility to enteritis, and these microbes could be

523 promoting clostridial infections by establishing a niche conducive to colonization in a yet-to-be 
524 determined manner. 4) Diet - including trypsin inhibitors - can also influence susceptibility to

525 clostridial enteritis. Although, commercial nectars were low in trypsin inhibitors, we cannot rule

526 out the possibility that other supplementary food items (e.g. sweet potatoes or legumes) could

527 have contributed to toxin-mediated enteritis. Clostridial enteritis, and C. perfringens in

528 particular, not only affects lorikeets, but can also cause devastating losses in the poultry industry

529 (commonly Type G C. perfringens with NetB toxin), and lead to gastrointestinal disease in

530 humans and other mammals - depending on the toxinotype. This work provides a foundation for

531 understanding gut microbiota features that are permissive to clostridial colonization and host

532 factors (e.g. age, prior infection) that shape responses to infection.

534 Methods

\section{Sample Collection - Columbus Zoo and Aquarium}

537 Cloacal swabs were obtained from all healthy lorikeets at the CZA ( $\mathrm{n}=67$ birds) during routine

538 flock health surveys at 3 timepoints (November 2018, February 2019, September 2019) (See

539 Additional File 1, a for experimental design). The flock was composed of four species of lories

540 and lorikeets including: rainbow lorikeets (Trichoglossus moluccanus), coconut lorikeets

541 (Trichoglossus haematodus), marigold lorikeets (Trichoglossus capistratus), and lorys

542 (Trichoglossus). Each bird was weighed and body condition scored during these surveys. Cloacal

543 swabs, intestinal tissue, and/or intestinal contents were collected opportunistically from lorikeets

544 ( $n=34$ birds) that presented with enteritis or died / were euthanized due to enteritis between

$545 \quad$ November 2018 and September 2019. 
547 A total of 246 samples were collected from birds at CZA - 172 samples from healthy birds and

54874 samples from birds with enteritis. Twenty-eight additional samples were also collected from

549 the environment and included samples of freshly prepared nectar, water, fruit, and swabs of the

550 aviary floor and perches. Swabs from live birds that presented with enteritis were collected prior

551 to initiation of antimicrobial therapy and, in some cases, throughout treatment. For necropsies

552 performed at CZA, intestinal contents were milked directly into a $2 \mathrm{ml}$ screw-top tube without

553 buffer. Swabs (Puritan, Guilford, ME) and intestinal contents were immediately transferred to a -

$55480^{\circ} \mathrm{C}$ freezer and stored until sample processing.

\section{Sample Collection - Denver Zoo}

557 Cloacal swabs were collected from the entire lorikeet flock during a flock survey in May 2019.

558 At this time, all lorikeets were reported to be healthy. Cloacal swabs, intestinal tissue, and

559 intestinal contents were collected opportunistically from lorikeets $(n=12)$ that died or were

560 euthanized due to enteritis between November 2018 and May 2019 (see Additional File 1, b for

561 experimental design). These birds were then age-, sex-, and species-matched to 12 healthy

562 lorikeets. Upon collection, all swabs and intestinal contents were immediately transferred to a -

$56380^{\circ} \mathrm{C}$ freezer and stored until sample processing and DNA extraction.

565 DNA extraction, 16S rRNA amplification, and sequencing

566 Bacterial DNA isolation from cloacal swabs and intestinal contents (approximately $200 \mathrm{mg}$ ) was

567 performed using QIAamp PowerFecal DNA Kits (Qiagen, Venlo, Netherlands). For cloacal

568 swabs, powerbeads and lysis buffer were added directly to the screw top tubes containing the

569 swabs. A bead beating step $(6 \mathrm{~m} / \mathrm{s}$ for $40 \mathrm{sec}$.) was used to replace the vortex step from the 
570 manufacturer's protocol. The remainder of the isolation was executed according to the protocol.

571 DNA isolation from formalin-fixed paraffin-embedded (FFPE) intestinal tissues collected at

572 necropsy was performed using the QIAamp DNA FFPE Tissue Kit following the manufacturer

573 recommendations. Following DNA isolation, DNA concentration was measured using a Qubit

574 Fluorometer 4 (Invitrogen, Carlsbad, CA, USA), and purity was assessed with a NanoDrop 1000

575 Spectrophotometer (Thermo Scientific, Waltham, MA, USA). Samples were submitted for

576 library preparation and sequencing at Argonne National Laboratory. Earth Microbiome Project

577 primers $515 \mathrm{~F}$ and $806 \mathrm{R}$ were used to amplify the V4 hypervariable region of the bacterial $16 \mathrm{~S}$

578 rRNA gene. Amplicons were sequenced on an Illumina MiSeq in 2 x 250 paired-end mode.

$580 \quad 16 S$ rRNA sequence processing

581 The 16S rRNA sequences were processed, filtered, and analyzed using QIIME 2 version 2020.11

582 [101] and DADA2 [102]. Taxonomic assignment of amplicon sequence variants (ASVs) was

583 performed using the Greenegenes 13_8 database with 99\% sequence identity cutoff. (Note: We

584 also performed taxonomic assignment with SILVA 132 and found that, in this case, Greengenes

585 provided more specific taxonomic assignments, particularly in the Clostridia taxa.) A total of 246

586 samples from CZA and 30 samples from DZ were submitted for sequencing. Samples with fewer

587 than 1000 reads were removed from analyses including 23 CZA samples and 6 DZ samples.

588 This resulted in a total of 223 CZA samples and 24 DZ samples that were used in our analyses.

589 After filtering, we obtained a total of 3,236,674 reads from the CZA samples (average: 13,911

590 reads per sample; range: 1003 to 68,537 reads) and 264,769 reads from the DZ samples (average:

5919,026 reads per sample; range: 1922 to 26,766 reads). Sequences identified as mitochondria,

592 chloroplasts, or eukaryotic reads were removed. Based on an examination of negative controls, 
593 we also identified the following taxa as contaminants and removed them from analyses: a taxa in

594 the order RF39 (Mollicutes phyla); a taxa in the genus Allobaculum, a taxa in the genus Massilia;

595 Haemophilus parainfluenzae; Prevotella copri; a taxa in the genus Sphingomonas; a taxa in the

596 genus Bradyrhizobium; Pseudomonas viridiflava; and a taxa in the genus Thermicanus.

598 Culture, bacterial identification, and genotyping

599 Lorikeet intestinal contents collected at necropsy were plated on reduced Trypticase Soy Agar

600 (TSA II) with 5\% sheep blood (BD BBL, Franklin Lakes, NJ) and incubated anaerobically (5\%

$\left.601 \mathrm{CO}_{2}, 5 \% \mathrm{H}_{2}, 90 \% \mathrm{~N}_{2}\right)$ at $37^{\circ} \mathrm{C}$ until growth was seen (24 - 48 hours). As needed, bacteria was

602 sub-cultured on C. perfringens selective agar, tryptose sulfite cycloserine (TSC), (Sigma Aldrich,

603 St. Louis, MO). Bacterial colony identification was performed via Matrix Assisted Laser

604 Desorption/Ionization Time-of-Flight Mass Spectrometry (MALDI-TOF). C. perfringens isolates

605 were submitted for toxinotyping via multiplex PCR at the Ohio Department of Agriculture

606 Animal Disease Diagnostic Laboratory (ODA ADDL). This PCR included primers for toxin

607 types $\mathrm{A}-\mathrm{E}(c p a, c p b, c p b 2$, cpe, etx, and iota A). The same primer sets and thermal cycler

608 parameters were used to analyze DNA extracted from FFPE tissues for comparison.

\section{CZA Lorikeets submitted to pathology}

611 Between 2015 - 2019, naturally deceased or humanely euthanized CZA lorikeets (due to severe

612 clinical disease) underwent a complete macroscopic postmortem examination necropsied by

613 veterinarians at the Columbus Zoo and Aquarium. Intestinal tracts were collected from lorikeets

614 with enteritis $(n=28)$ and with unremarkable intestines $(n=7)$. Various organs, including the

615 intestinal tracts, were placed in $10 \%$ neutral buffered formalin for fixation, stored at room 
temperature, and submitted to The Ohio State University College of Veterinary Medicine for

617 evaluation of the formalin fixed organs and histopathology.

618

\section{Histopathology}

620 Intestinal tracts, as well as other collected and formalin fixed tissues, were routinely trimmed,

621 paraffin embedded, and stained with hematoxylin and eosin (H\&E) for initial evaluation by the

622 Comparative Pathology \& Digital Imaging Shared Resource (CPDISR). Intestines were

623 evaluated by two veterinary pathologists board-certified by the American College of Veterinary

624 Pathologists (FU and MM) to characterize cases as necrotizing enteritis (n=28) vs

625 control/unremarkable intestines $(n=7)$. All sections with necrotizing enteritis were subsequently

626 stained with the modified Brown-Hopps gram stain applied to identify and characterize

627 intralesional bacteria, and a Masson's Trichrome stain to characterize chronicity of the lesions

628 through the presence of fibrosis.

629

630 Immunohistochemistry

631 We then looked for the presence of Clostridium perfringens within intestinal sections from

632 lorikeets with $(n=28)$ or without enteritis $(n=7)$ via immunohistochemistry (IHC) using a

633 polyclonal rabbit anti-Clostridium perfringens antibody, OASA07164, Aviva Systems Biology,

634 San Diego, CA). The IHC protocol is described in Additional File 2.

635

Clostridium perfringens toxinotyping

637 Toxinotyping on DNA extracted from FFPE lorikeet intestinal tissues was performed at the San

638 Bernardino branch of the California Animal Health and Food Safety (CAHFS) Laboratory, 
639 University of California-Davis, using a previously established method[47]. Additional testing for

640 the $C$. perfringens alpha toxin (cpa) and beta-2 toxin (cpb2) was performed on CZA intestinal

641 isolates at The Ohio State University using the following primers: cpaF (5'-

642 GCTAATGTTACTGCCGTTGA -3'), cpaR (5'- CCTCTGATACATCG GTAAG -3'), cpb2F

643 (5' - AGATTTTAAATATGATCCTAACC -3') and cpb2R (5'-

644 CAATACCCTTCACCAAATACTC -3'). PCR conditions for $c p a$ and $c p b 2$ testing were as

645 follows: PCR was performed in a total volume of $25 \mu \mathrm{L}$ containing $0.75 \mu \mathrm{L}$ of each primer $(0.3$

$646 \mu \mathrm{M}), 2 \mu \mathrm{L}$ of extracted DNA, $12.5 \mu \mathrm{L}$ of HiFi Hot Start Master Mix (Kapa Biosystems) and 9

$647 \mu \mathrm{L}$ of nuclease-free water. Thermocycler profiles were as follows: $95{ }^{\circ} \mathrm{C}$ for $3 \mathrm{~min}, 45$ cycles of

$64895{ }^{\circ} \mathrm{C}$ for $30 \mathrm{~s}, 55^{\circ} \mathrm{C}$ for $45 \mathrm{~s}$, and $72{ }^{\circ} \mathrm{C}$ for $60 \mathrm{~s}$, and a final extension step at $72{ }^{\circ} \mathrm{C}$ for $5 \mathrm{~min}$.

649 Positive controls included DNA extracted from known, toxin typed, C. perfringens isolates.

650

651 Clostridium colinum PCR

652 PCR for C. colinum was also performed at CAHFS. Scrolls from (FFPE) sections of small

653 intestine from the lorikeets were deparaffinized and the DNA extracted using a commercial kit

654 (QIAamp DNA FFPE tissue kit; Qiagen) following the manufacturer's instructions. The

655 extracted DNA was used as a template for PCR amplification of a 192-bp fragment of the 16S

656 rRNA gene of $C$. colinum, using the primers CcolF (5'- CGGCTGGATCACCTCCTTTC-3') and

657 CcolR (5'-ACATTTTTGTCTGGCTCACGA-3'). PCR was performed in a total volume of $25 \mu \mathrm{L}$

658 containing $0.5 \mu \mathrm{L}$ of each primer $(0.5 \mu \mathrm{M}), 3 \mu \mathrm{L}$ of extracted DNA, $7 \mu \mathrm{L}$ of nuclease-free water, 659 and $14 \mu \mathrm{L}$ of Platinum ${ }^{\mathrm{TM}}$ II Hot-Start Green PCR Master Mix (2X) (Invitrogen). Thermocycler 660 profiles were as follows: $95^{\circ} \mathrm{C}$ for $7 \mathrm{~min}, 35$ cycles of $95^{\circ} \mathrm{C}$ for $60 \mathrm{~s}, 60{ }^{\circ} \mathrm{C}$ for $60 \mathrm{~s}$, and $72{ }^{\circ} \mathrm{C}$

661 for $60 \mathrm{~s}$, and a final extension step at $72{ }^{\circ} \mathrm{C}$ for $7 \mathrm{~min}$. Samples were held at $4{ }^{\circ} \mathrm{C}$. Positive 
662 controls included DNA extracted from a commercial bacterial strain (ATCC 27770) and from

663 FFPE sections of quail disease cases in which C. colinum had been isolated. DNA extracted from

664 DZ samples also underwent C. colinum PCR.

665

666 Measurement of trypsin inhibitor levels in nectar

667 We measured trypsin inhibitor levels in six commercial lorikeet feeds commonly used at

668 zoological institutions and aviaries around North America, including at CZA and DZ. Lorikeet

669 feeds included: Mazuri Softbill Diet for Iron-Sensitive Birds (Mazuri Exotic Animal Nutrition,

670 St. Louis, MO), Blessing's Gourmet Blend Lory Nectar (Blessing's Pet Food Products, Murrieta,

671 CA), Mazuri Lorikeet Diet (Formula: 5AB4, Mazuri Exotic Animal Nutrition, St. Louis, MO),

672 Rainbow Landing Nectar (Berwick Productions, Inc. Escondido, CA), Roudybush Lory Nectar

67315 (Roudybush, Woodland, CA), and Higgins Intune Lory Food (Higgins Premium Pet Foods,

674 Miami, FL).

675

676 Nectar preparation

677 To eliminate assay interference due to free fatty acids, all nectars were first defatted through a

678 hexane (Thermo Fisher Scientific, Waltham, MA) extraction. Nectars were combined with three

679 times their volume of pure hexane and mixed for one minute. The samples were then allowed to

680 sit for 10 minutes to allow for a separation of layers, and the top hexane-fat layer was removed.

681 This process was repeated a total of three times for each nectar. Defatted nectars were then

682 allowed to dry overnight in a fume hood. Once dry, $1 \mathrm{~g}$ of defatted nectar was added to $50 \mathrm{~g}$ of

$6830.01 \mathrm{M} \mathrm{NaOH}$ (Thermo Fisher Scientific, Waltham, MA). The mixture was stirred slowly on a 
684 stir plate for 3 hours. Extracts were then centrifuged at $4696 \mathrm{x} \mathrm{g}$, and the supernatant was

685 decanted to produce the final extract.

687 Trypsin inhibitor assay

688 Trypsin inhibitor assays were carried out based on standard American Association of Cereal

689 Chemists (AACC) methods [103], with modifications as proposed by Liu (2019) [40]. Reagent

690 preparation is described in Additional File 3. Nectar solutions were diluted to various levels by

691 combining $0-1 \mathrm{~mL}$ of nectar extract with enough deionized water to yield $2 \mathrm{~mL}$ total.

692 Concentration ranges chosen were based upon preliminary trials, with the aim of yielding

693 absorbance data points that fell in the range of $30-70 \%$ inhibition. The $2 \mathrm{~mL}$ of diluted nectar

694 were added to 15 -mL centrifuge tubes and combined with $5 \mathrm{~mL}$ benzoyl-DL-arginine-p-

695 nitroanilide hydrochloride (BAPA) solution. The mixture was incubated in a $37^{\circ} \mathrm{C}$ water bath to

696 bring it up to temperature, and then the assay reaction was initiated by adding $2 \mathrm{~mL}$ pre-warmed

697 trypsin solution $(0.02 \mathrm{mg}$ trypsin/mL) to each tube. The tubes were allowed to react for exactly

69810 minutes at $37^{\circ} \mathrm{C}$, then the reaction was stopped with the addition of $1 \mathrm{~mL} 30 \%$ acetic acid

699 solution. Samples were allowed to cool to room temperature before measuring absorbance at 410

700 nm with an HP 8453 UV-Vis spectrophotometer (Hewlett Packard, Palo Alto, CA). Absorbance

701 readings were corrected with nectar blanks by mixing all reagents, but adding trypsin solution

702 after the acetic acid to ensure the enzyme was inactive. A positive control sample was also made

703 using $2 \mathrm{~mL}$ water in place of nectar and running the assay as delineated above.

704

705

TIU calculation 
706 With the definition that $1 \mathrm{TIU}=$ a decrease in 0.01 absorbance compared to a positive control

707 sample, TIU/mg could be calculated as follows:

708

$$
\text { TIU }=(\text { positive control sample absorbance }- \text { nectar sample absorbance }) / 0.01
$$

$$
\mathrm{TIU} / \mathrm{mg}=\mathrm{TIU} /[\mathrm{Nectar} \text { concentration }(\mathrm{mg} / \mathrm{mL}) \times 10 \mathrm{~mL} \text { assay solution }]
$$

To compare trypsin inhibitor concentrations (TIU/mg) between nectars, we applied a one-way

711 ANOVA followed by pairwise Tukey’s tests.

712

\section{Statistical analyses}

714 We compared the number of lorikeets that ever had enteritis versus the number of lorikeets that

715 never developed enteritis by age, sex, and species using a $\chi^{2}$ test [104]. In cases where groups

716 had a frequency less than 5, we use the Yates' $\chi^{2}$ correction. For cases in which a group

717 contained zero individuals (e.g. 0 females), we used the Freeman-Halton extension of the

718 Fisher's exact test. To compare average age across groups, we used a Kruskal-Wallis test after

719 testing for normality using a Shapiro-Wilk test. For microbial community analyses, alpha

720 diversity was compared between groups using observed features, the Shannon diversity metric,

721 and the Kruskal-Wallis test. Beta diversity was evaluated using permutational multivariate

722 analysis of variances (PERMANOVAs) between groups on Bray-Curtis distance matrices. All

723 alpha and beta diversity $p$-values were corrected for multiple comparisons using the Benjamini

724 Hochberg false discovery rate (FDR) correction. A $p$-value $<0.05$ was considered significant.

725 Differential abundances of microbes by status (healthy, enteritis, susceptible) or season

726 (November 2018, February 2019, September 2019) were tested using an ANCOM [105]. 
728 Machine learning methods were used to assess susceptibility to enteritis based on microbial

729 relative abundances and other demographic factors. Specifically, microbial composition was

730 used in a supervised setting for classifying birds into True Healthy and Susceptible groups. True

731 Healthy birds never developed enteritis throughout the sampling period (Nov. 2018 - Sept. 2019)

732 while Susceptible birds developed enteritis at least once during this time. Random forests

733 (RF)[106] and logistic regression with appropriate regularization (LASSO)[107, 108] were

734 employed to differentiate these groups. The predictive accuracy was then assessed through cross-

735 validation using an area under the receiver-operating characteristics curve (ROC).

736

\section{Declarations}

738 Ethics approval and consent to participate

739 IACUC approval through The Ohio State University (\# 2019A00000028).

740 Consent for publication

741 Not applicable.

742 Availability of data and material

743 Sequencing data is available at NCBI Bioproject PRJNA722436.

$744 \quad$ Competing Interests

745 Not applicable.

$746 \quad$ Funding

747 Funding for this project provided by the following:

748 Columbus Zoo/Ohio State Cooperative Grants Program

749 National Institutes of Health Training Grant (T35)

750 The Ohio State University Infectious Diseases Institute 
751 The Ohio State University College of Veterinary Medicine

752 American Association of Zoo Veterinarians' Wild Animal Health Fund

753 The Comparative Pathology \& Digital Imaging Shared Resource is supported in part by grant

754 P30 CA016058 from the National Cancer Institute, Bethesda, MD.

755

756 Authors' contributions

$757 \mathrm{RJ}$ and $\mathrm{JH}$ were involved in identification of the clinical syndrome, project conceptualization,

758 sample collection, and clinical data.

759 DM, CM, and VH were involved in project development, DNA extraction, sequencing data

760 analysis and interpretation, manuscript writing, and figure preparation.

761 VH also obtained project support and oversaw/managed the project and performed demographic

762 / epidemiological analyses. RMrochak, RMadan, KS, and CM were involved in toxinotyping on

763 intestinal isolates. ME was involved in sequencing data filtering and processing.

764 LG and SP were involved in Random Forests and LASSO analysis and interpretation, manuscript

765 writing, and figure preparation. KF-K, MM, KLP, GB, and FU were involved in histopathology

766 analysis, staining, and interpretation. $\mathrm{MM}$ and FU were also involved in manuscript writing and

767 figure preparation. KF-K and MM were involved in gross pathology interpretation and figure

768 preparation. FU performed histopathology, IHC analysis, interpretation, and figure preparation.

769 MN performed toxinotyping on FFPE blocks, C. colinum PCR, and was involved in manuscript

770 writing and table preparation. WC, KS, and YV were involved in trypsin inhibitor testing and

771 data interpretation. WC and YV were also involved manuscript writing and figure preparation.

772

773 Acknowledgements 
774 We would like to acknowledge all of the keepers and veterinary staff at the Columbus Zoo and

775 Aquarium and the Denver Zoo for facilitating sample collection and providing detailed health,

776 medication, and dietary record. We also thank Dr. Michael Martinez for assistance with gross

777 pathology photos, and we gratefully acknowledge Drs. Tamara Kruse and Helena Mendes-

778 Soares for early discussions that shaped this project.

779

780

Figure Legends

781

782

Figure 1. Seasonality in enteritis and gut microbiota in healthy lorikeets a) Number of

enteritis cases by month in Columbus Zoo and Aquarium lorikeets. Enteritis samples collected

from the same bird within two weeks of the initial enteritis sample were considered a single case.

Enteritis samples collected beyond 2 weeks from the initial sample in the same bird were

counted as a second case of enteritis. Columbus Zoo \& Aquarium healthy birds only: b)

787 Microbial community composition (Weighted UniFrac) and c) diversity (Observed Features) by

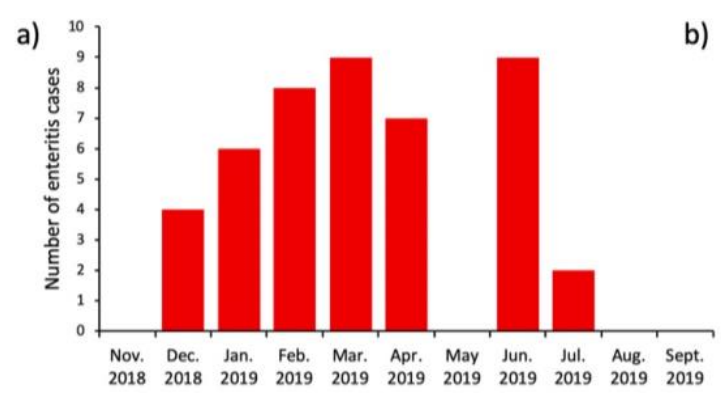

b)

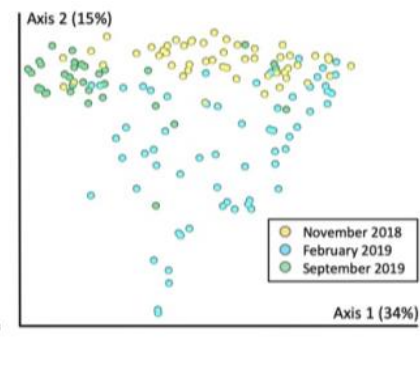

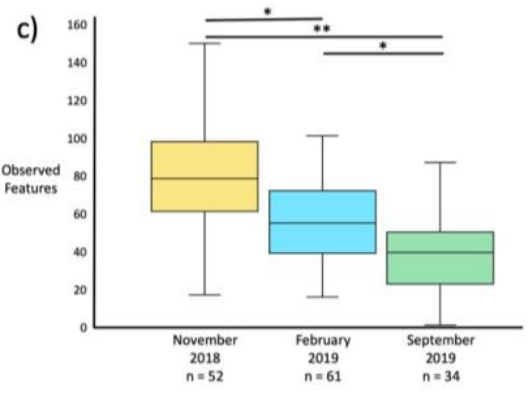


Figure 2. Microbial community analysis in CZA lorikeets with enteritis. Microbial

795

796

797

798

799

800

801

802

803

804

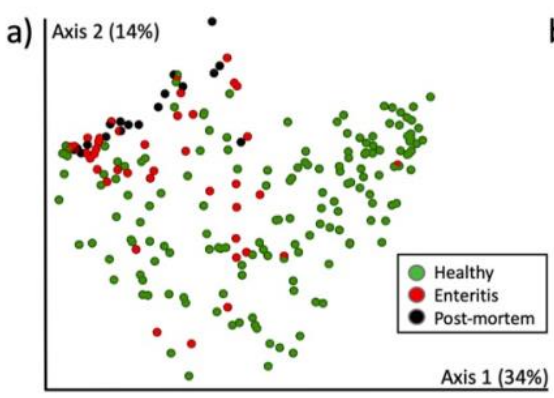

805

806

807

808

809

810

811

812

had both enteritis and healthy samples.

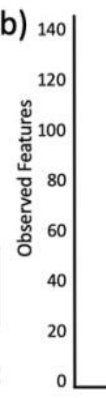

composition and diversity in healthy lorikeets, lorikeets with enteritis, and lorikeets that died or were euthanized due to enteritis (postmortem). a) Microbial composition (Weighted UniFrac)

was significantly altered (PERMANOVA $p=0.001)$ and $\mathbf{b}$ ) microbial diversity (Observed

Features) was significantly decreased (Kruskal-Wallis $* p<0.0005, * * p<1 \times 10^{-9}$ ) in lorikeets

with enteritis or postmortem lorikeets. (Also see Additional File 7.) c) The relative abundance of

Clostridium perfringens was significantly increased lorikeets with enteritis across all birds

(ANCOM, $\mathrm{W}=1098)$ and across matched birds (ANCOM, W = 788). "All birds" included

samples from healthy birds that never got enteritis. "Matched birds" included only 25 birds that

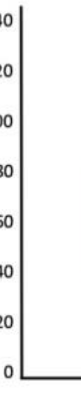

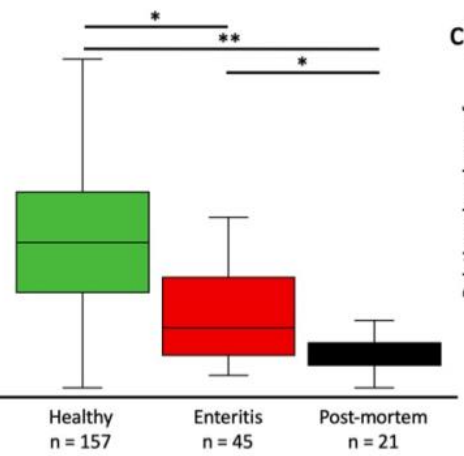

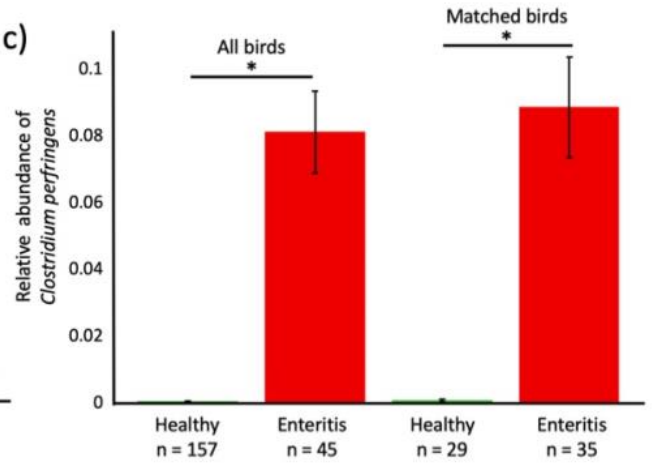

Figure 3. Lorikeet enteritis gross and histopathology. a) Number of lorikeet enteritis versus

non-enteritis deaths by month. Cases from CZA were diagnosed histologically over a 5-year period (2015-2019). An increase in enteritis cases were observed during the summer months (June-September). (b-d) Gross necropsy findings typical of lorikeets with enteritis. b) The feathers have been plucked to demonstrate the decreased pectoral muscle mass of affected birds leading to a prominent keel. c) There are multiple severely dilated loops of intestines, including the paired ceca, with thin intestinal walls. Also present are segments of thickened and nodular 
813 intestines mottled dark tan and pale tan. Tissues are formalin fixed. d) Formalin fixed loop of

814 small intestine from an affected lorikeet. There are multiple soft to firm pale tan nodules within

815 the wall and on the serosa of the intestines. The mesentery is also thickened by pale tan tissue

816 and similar nodules. (e-g) Histologic findings from lorikeets with enteritis. e) Hematoxylin and

817 eosin stain (H\&E) of a chronic case of necrotizing enteritis in a lorikeet. Two loops of intestine

818 are markedly dilated with thinning of the intestinal wall and replacement with fibrosis. The

819 lumens are impacted with a coagulum of degenerate red blood cells, bacterial colonies and

820 sloughed mucosa that often compress the remaining atrophied and blunted intestinal villi. There

821 is also inflammation on the serosa and adjacent mesentery. f) H\&E of acute transmural

822 necrotizing and ulcerative enteritis in a lorikeet. The sparse remaining mucosa is characterized

823 by a large central ulcer, inflammatory cells including macrophages and heterophils throughout

824 the intestinal wall centered around the ulcer and overlying large colonies of bacteria. g) Modified

825 Brown-Hopps of same intestinal section as in Fig 3f. The superficially adhered bacterial colonies

826 within the lesion are monomorphic large gram-positive bacilli. h) H\&E of necrotizing and

827 ulcerative enteritis in a lorikeet. There is little remaining mucosa with a large central focus of

828 ulceration, numerous heterophils and macrophages, followed by a layer of fibrin and degenerate

829 red blood cells with admixed large bacterial colonies and sloughed necrotic mucosal epithelium.

830 i) Immunohistochemistry (IHC) against Clostridium perfringens from the same intestinal section

831 as in Fig 3h. Box indicates region in Fig 3j under higher magnification. j) IHC against $C$.

832 perfringens within the indicated region from Fig3i. The light brown staining is non-specific

833 labeling of the sloughed necrotic mucosa and hemorrhage. The punctate dark brown staining

834 indicates immunolabeling of bacteria within the necrohemorrhagic coagulum and focus of

835 ulceration. 

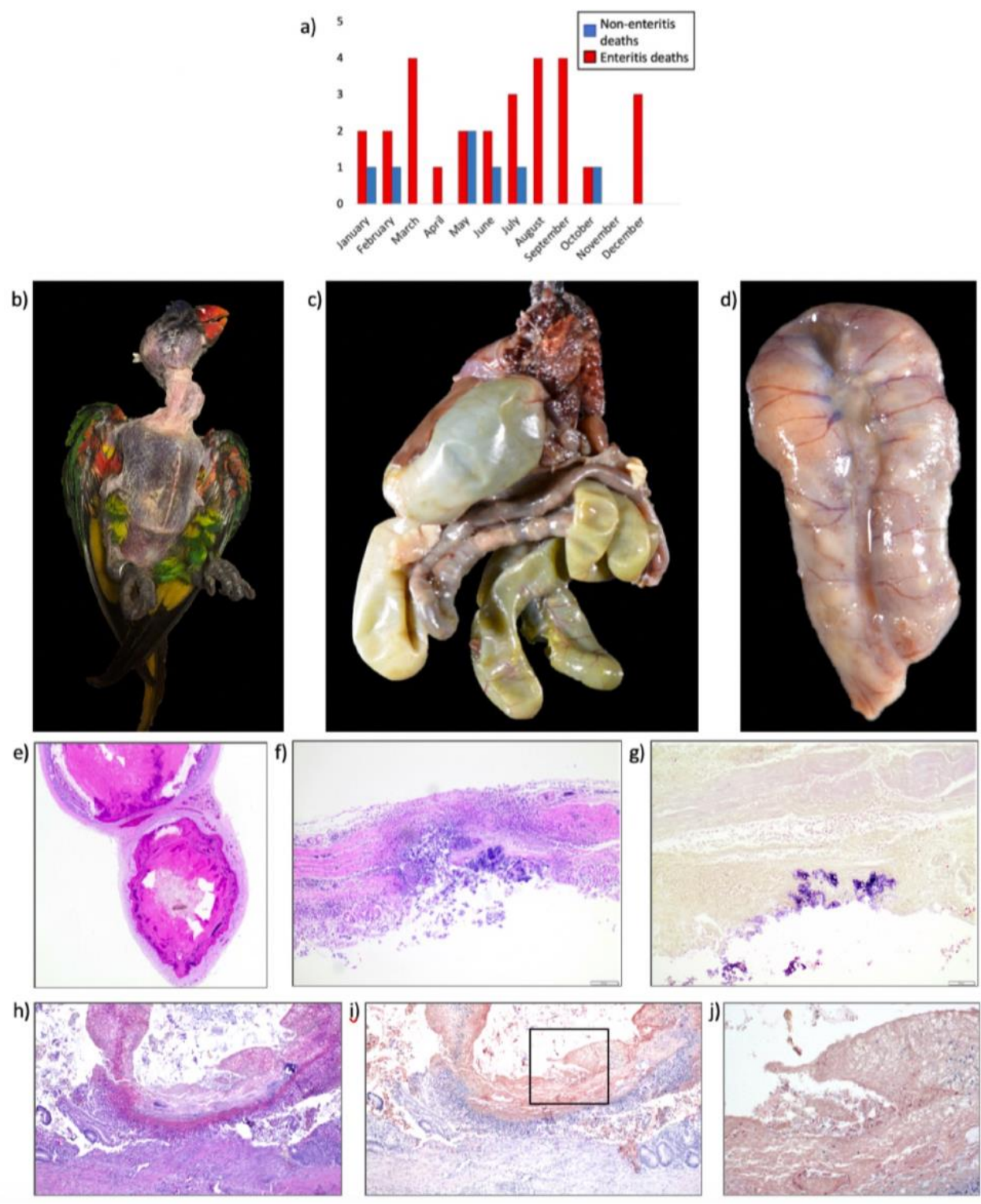

Figure 4. Altered microbial community diversity and composition in Denver Zoo (DZ)

lorikeets with enteritis. Microbial composition and diversity in healthy lorikeets and lorikeets that died or were euthanized due to enteritis ("post-mortem” lorikeets). a) Microbial composition (Weighted UniFrac) was significantly altered (PERMANOVA $p=0.001$ ) and b) microbial diversity (Observed Features) was significantly decreased (Kruskal-Wallis $* p<0.0005$ ) in postmortem lorikeets. (Also see Additional File 11.) c) The relative abundance of $C$. perfringens 
844 was also significantly increased in post-mortem lorikeets as compared to healthy lorikeets

845 (ANCOM, W = 67). d) Taxa bar plots showing taxonomic distributions within healthy and post-

846 mortem lorikeets with enteritis. Post-mortem lorikeet microbial communities were dominated by

847 C. perfringens.
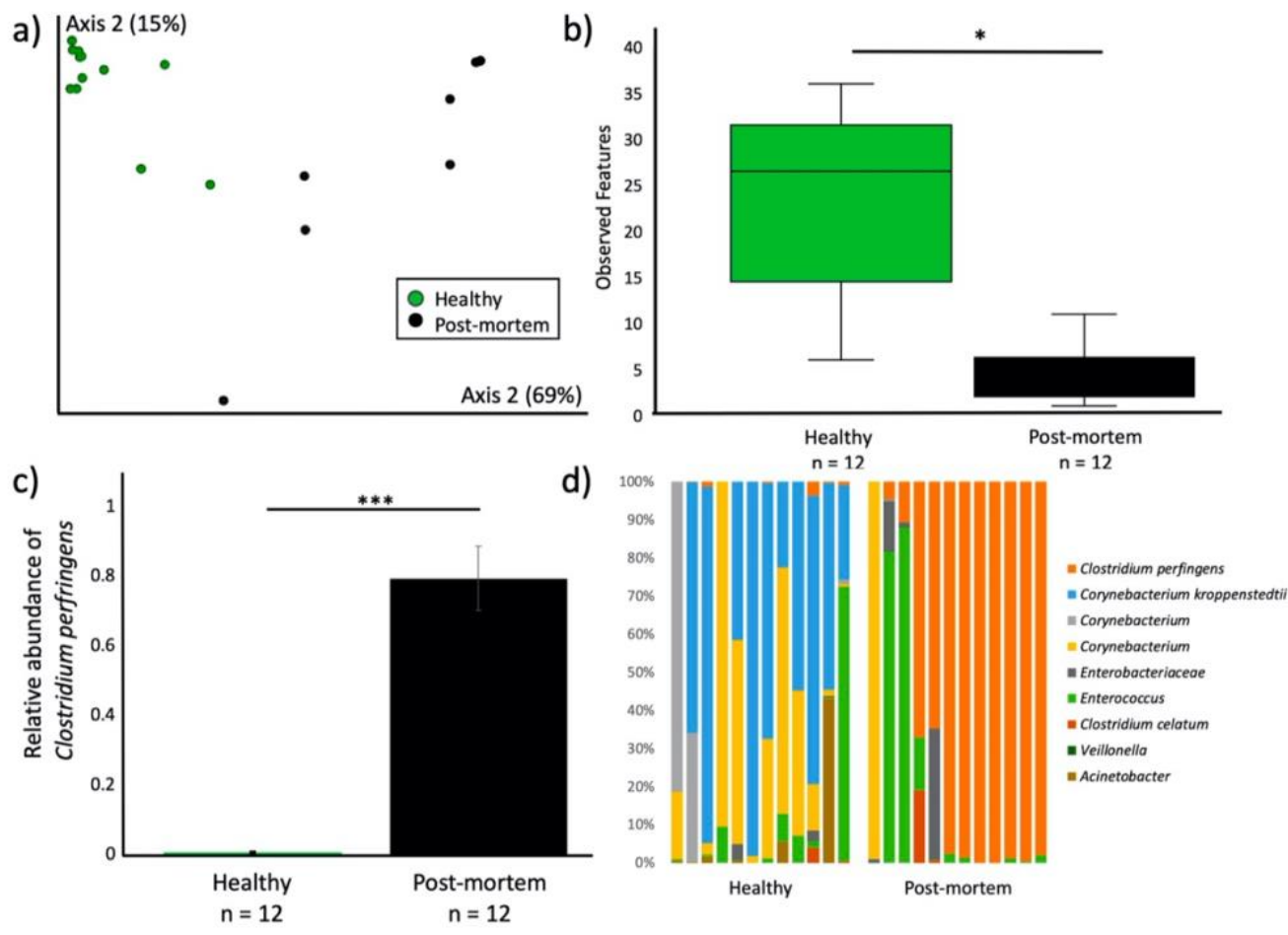

851 microbial composition that predicts enteritis. Healthy lorikeets that never developed enteritis

852 throughout the sampling period were identified as "True Healthy" while healthy birds that

853 developed enteritis at least once during the sampling period were identified as "Susceptible."

854 "Enteritis" represents birds with enteritis that were sampled while they were clinically ill. No

855 post-mortem samples are included in this figure. a) Microbial diversity (Observed Features,

856 Kruskal-Wallis, all $p<0.005)$ was increased and b) microbial composition was altered 
857 (PERMANOVA $p=0.001$ ) in Susceptible birds. c) Twenty-six variables including 24 microbial

858 taxa and two demographic variables were identified in Random Forests and LASSO models as

859 predictive of susceptibility or true health. The size of the bars represents the effect size of each

860 variable as predicted by the RF model. (Also see Additional File 12.)

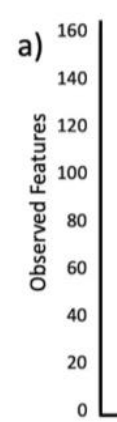

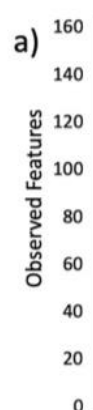

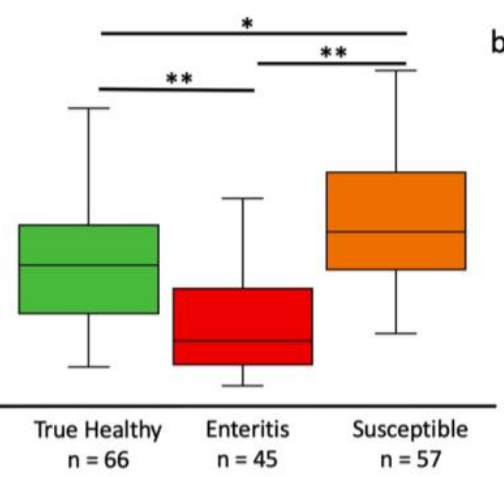
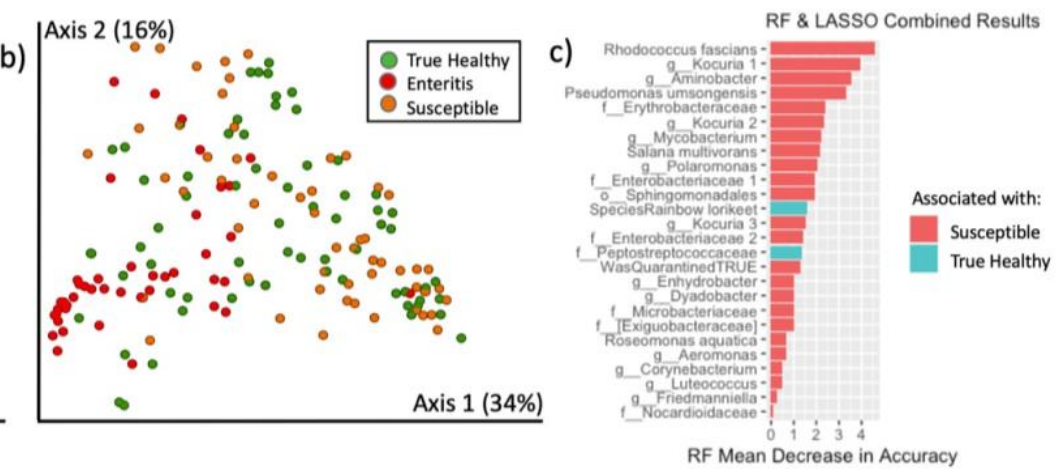

862

Figure 6. Trypsin inhibitor measurements in commercial nectars. Trypsin inhibitor units significantly different.

2.5

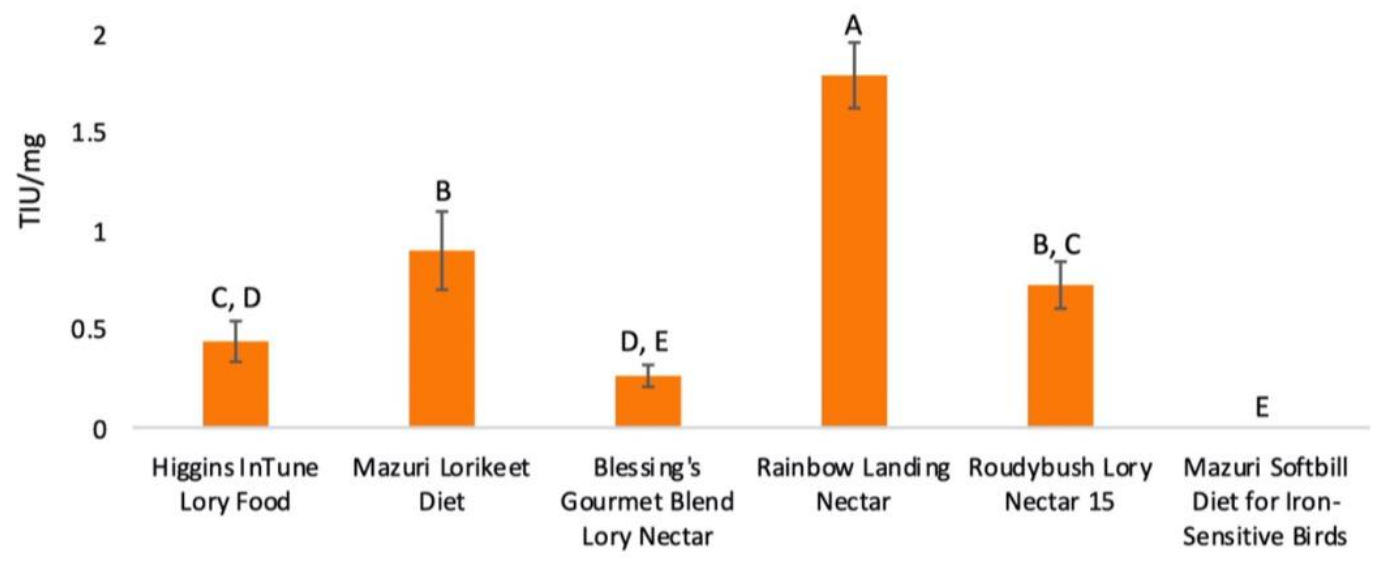


871 Table 1. Columbus Zoo and Aquarium (CZA) Lorikeet Demographics. Number and percent

872 of Columbus Zoo \& Aquarium lorikeets that ever or never had enteritis between Nov. 2018 and

873 Sept. 2019 by sex, age, and lorikeet species.

Table 1. Columbus Zoo and Aquarium (CZA) Lorikeet Demographics.

\begin{tabular}{|c|c|c|c|}
\hline & $\begin{array}{l}\text { Lorikeets that had } \\
\text { one or more } \\
\text { episodes of } \\
\text { enteritis between } \\
\text { Nov. 2018-Sept. } \\
2019 \\
\end{array}$ & $\begin{array}{c}\text { Lorikeets that } \\
\text { never had enteritis } \\
\text { between } \\
\text { Nov. 2018-Sept. } \\
2019\end{array}$ & $p$-value \\
\hline $\operatorname{Sex}(n, \%)$ & & & \multirow{4}{*}{$\begin{array}{c}\mathrm{p}=0.06 \text { (all) } \\
\mathrm{p}=0.73 \text { (male } \\
\text { vs. female } \\
\text { only) }\end{array}$} \\
\hline Male & $9(13.4 \%)$ & 14 (20.9\%) & \\
\hline Female & $11(16.4 \%)$ & $14(20.9 \%)$ & \\
\hline Unknown & $14(20.9 \%)$ & $5(7.5 \%)$ & \\
\hline \multicolumn{4}{|l|}{ Age (n, \%) } \\
\hline $0-2$ years & $19(28.3 \%)$ & $7(10.4 \%)$ & \multirow{4}{*}{$p=0.02$} \\
\hline $3-5$ years & $7(10.4 \%)$ & $16(23.9 \%)$ & \\
\hline $6-10$ years & $6(9.0 \%)$ & $6(9.0 \%)$ & \\
\hline$>11$ years & $2(3.0 \%)$ & $4(6.0 \%)$ & \\
\hline \multicolumn{4}{|c|}{ Species (n, \%) } \\
\hline Rainbow & $8(11.9 \%)$ & $17(25.4 \%)$ & \multirow{4}{*}{$\begin{array}{c}p=0.07 \text { (all) } \\
p=0.01 \\
\text { (rainbow vs. } \\
\text { coconut only) }\end{array}$} \\
\hline Coconut & $23(34.3 \%)$ & 13 (19.4 \%) & \\
\hline Marigold & $1(1.5 \%)$ & $0(0 \%)$ & \\
\hline Lory & $2(3 \%)$ & $3(4.5 \%)$ & \\
\hline
\end{tabular}


Table 2. Intestinal content isolates from CZA lorikeets

\begin{tabular}{|c|c|c|c|c|c|c|}
\hline Bird ID & $\begin{array}{l}\text { Intestinal } \\
\text { contents, } \\
\text { growth in } \\
\text { culture? }\end{array}$ & MALDI identification & $\begin{array}{l}\text { cpa } \\
\text { (alpha } \\
\text { toxin) }\end{array}$ & $\begin{array}{l}\text { cpb2 } \\
\text { (beta2 } \\
\text { toxin) }\end{array}$ & $\begin{array}{l}\text { Inferred } \\
\text { C. perfringens } \\
\text { toxinotype }\end{array}$ & $\begin{array}{c}\text { Selected for } \\
\text { susceptibility } \\
\text { testing? }\end{array}$ \\
\hline 104073 & No & None & & & & \\
\hline 113096 & Yes & $\begin{array}{l}\text { Clostridium perfringens and } \\
\text { Clostridium disporicum }\end{array}$ & + & + & A & \\
\hline 115057 & No & None & & & & \\
\hline 115069 & Yes & Enterococcus faecium & & & & \\
\hline 115094 & No & None & & & & \\
\hline 116046 & Yes & Esherichia coli & & & & \\
\hline 118064 & Yes & Clostridium perfringens & + & + & A & \\
\hline 118071 & Yes & $\begin{array}{l}\text { Clostridium perfringens, } \\
\text { Escherichia coli, and } \\
\text { Clostridum paraperfringens } \\
\text { or Clostridum baratii }\end{array}$ & + & + & A & yes \\
\hline 118075 & Yes & Clostridium perfringens & + & + & A & yes \\
\hline 118089 & Yes & $\begin{array}{l}\text { Clostridium perfringens, } \\
\text { Esherichia coli, an organism } \\
\text { with no ID }\end{array}$ & + & + & A & \\
\hline 118115 & No & None & & & & \\
\hline 118118 & Yes & $\begin{array}{l}\text { Clostridium perfringens and } \\
\text { Clostridium disporicum }\end{array}$ & + & - & A & \\
\hline 118119 & Yes & Clostridium paraputrificum & & & & \\
\hline
\end{tabular}

879 Table 3. Lorikeet Demographics for CZA lorikeets submitted to pathology. The Freeman-

880 Halton extension of the Fisher's Exact test was used to calculate p-values for sex and species. A

881 Kruskal-Wallis test was used to calculate the p-value for age.

Table 3. Lorikeet Demographics for CZA lorikeets submitted to pathology

\begin{tabular}{|lccc|}
\hline & $\begin{array}{c}\text { Lorikeets with } \\
\text { enteritis }\end{array}$ & $\begin{array}{c}\text { Lorikeets with } \\
\text { no enteritis }\end{array}$ & p-value \\
\hline Sex (n, \%) & & & $p=0.73$ (all) \\
Male & $12(34.3 \%)$ & $5(14.3 \%)$ & $\begin{array}{c}p=0.06 \\
\text { (male vs. } \\
\text { Female }\end{array}$ \\
Unknown & $12(34.3 \%)$ & $0(0 \%)$ & female only) \\
\hline Age (Mean \pm SD) & $4.6 \pm 4.7$ & $9.3 \pm 6.1$ & $p=0.13$ \\
\hline Species (n, \%) & & & \\
Rainbow & $7(20 \%)$ & $5(14.4 \%)$ & \\
Coconut & $14(40 \%)$ & $1(2.9 \%)$ & $p=0.11$ \\
Other & $7(20 \%)$ & $1(2.9 \%)$ & \\
\hline
\end{tabular}


bioRxiv preprint doi: https://doi.org/10.1101/2021.09.08.459483; this version posted September 8, 2021. The copyright holder for this preprint (which was not certified by peer review) is the author/funder, who has granted bioRxiv a license to display the preprint in perpetuity. It is made available under aCC-BY 4.0 International license.

Table 4. Toxin profiles, IHC, and $C$. colinum PCR on lorikeet intestines submitted to

pathology

Table 4. Toxin profiles, IHC, and C. colinum PCR on lorikeet intestines submitted to pathology

\begin{tabular}{|c|c|c|c|c|c|c|c|c|c|c|c|c|c|}
\hline Sample ID & Age (yrs) & Sex & Species & $\begin{array}{c}\text { Acute / } \\
\text { Chronic }\end{array}$ & Status & $\begin{array}{c}\text { C. } \\
\text { perfringens } \\
\text { IHC }\end{array}$ & $c p a$ & $c p b$ & etx & $i t x$ & cpe & NetB & $\begin{array}{c}\text { C. } \\
\text { colinum } \\
\text { PCR }\end{array}$ \\
\hline $15-1444$ & 3 & $\mathrm{~F}$ & Rainbow & acute & enteritis & + & - & - & - & - & - & - & + \\
\hline $15-1455$ & 14.7 & $\mathrm{~F}$ & Rainbow & acute & enteritis & + & + & - & - & - & - & - & + \\
\hline $15-1456$ & 5.4 & $\mathrm{M}$ & Coconut & acute & enteritis & - & + & - & - & - & - & - & + \\
\hline $15-1636$ & 11 & $M$ & Rainbow & acute & enteritis & + & - & - & - & - & - & - & + \\
\hline $15-2050$ & 0.8 & $\mathrm{~F}$ & Lorikeet & acute & enteritis & - & - & - & - & - & - & - & - \\
\hline $18-1004$ & 0.8 & $\mathrm{~F}$ & Coconut & acute & enteritis & + & + & - & - & - & - & - & + \\
\hline $18-1304$ & 6 & $\mathrm{M}$ & Rainbow & acute & enteritis & + & - & - & - & - & - & - & - \\
\hline $18-1442$ & 2 & $M$ & Rainbow & acute & enteritis & + & + & - & - & - & - & - & + \\
\hline $18-1698$ & 15.5 & $\mathrm{~F}$ & Coconut & acute & enteritis & + & + & - & - & - & - & - & + \\
\hline $18-950$ & 2.1 & $\mathrm{~F}$ & Lory & acute & enteritis & + & - & - & - & - & - & - & - \\
\hline 19-1164 & 1 & $M$ & Coconut & acute & enteritis & - & - & - & - & - & - & - & + \\
\hline $19-1220$ & 1.3 & $\mathrm{~F}$ & Coconut & acute & enteritis & + & + & - & - & - & - & - & - \\
\hline $19-15$ & 8.6 & $\mathrm{~F}$ & Coconut & acute & enteritis & + & - & - & - & - & - & - & - \\
\hline $19-183$ & 0.8 & $\mathrm{~F}$ & Coconut & acute & enteritis & + & + & - & - & - & - & - & - \\
\hline $19-185$ & 3.9 & $\mathrm{~F}$ & Coconut & acute & enteritis & + & - & - & - & - & - & - & - \\
\hline $19-427$ & 0.6 & unk & Lorikeet & acute & enteritis & + & + & - & - & - & - & - & + \\
\hline $19-428$ & 0.9 & unk & Coconut & acute & enteritis & + & + & - & - & - & - & - & + \\
\hline $19-488$ & 15.6 & $\mathrm{M}$ & Rainbow & acute & enteritis & - & - & - & - & - & - & - & + \\
\hline $19-526$ & 1 & unk & Coconut & acute & enteritis & no tissue & - & - & - & - & - & - & - \\
\hline 15-1681 & 4.8 & $\mathrm{M}$ & Coconut & chronic & enteritis & + & + & - & - & - & - & - & + \\
\hline $15-1834$ & 8.9 & $\mathrm{M}$ & Lorikeet & chronic & enteritis & - & - & - & - & - & - & - & + \\
\hline 16-82 & 7 & $M$ & Lorikeet & chronic & enteritis & + & + & - & - & - & - & - & + \\
\hline $18-1561$ & 2.8 & M & $\begin{array}{l}\text { Violet } \\
\text { necked }\end{array}$ & chronic & enteritis & + & - & - & - & - & - & - & + \\
\hline 19-1241 & 1.3 & unk & Coconut & chronic & enteritis & + & + & - & - & - & - & - & + \\
\hline $19-1242$ & 3 & $\mathrm{~F}$ & Marigold & chronic & enteritis & + & + & - & - & - & - & - & - \\
\hline $19-863$ & 5 & $\mathrm{M}$ & Rainbow & chronic & enteritis & + & - & - & - & - & - & - & - \\
\hline $15-1228$ & 0.2 & M & Coconut & $\begin{array}{c}\text { chronic } \\
\text { active }\end{array}$ & enteritis & + & - & - & - & - & - & - & + \\
\hline $15-1230$ & 0.2 & $F$ & Coconut & $\begin{array}{c}\text { chronic } \\
\text { active }\end{array}$ & enteritis & + & - & - & - & - & - & - & + \\
\hline 15-1226 & 11 & $M$ & Rainbow & NA & No lesions & + & + & - & - & - & - & - & - \\
\hline $16-426$ & 0.2 & $\mathrm{M}$ & Lorikeet & NA & No lesions & - & - & - & - & - & - & - & - \\
\hline $16-653$ & 1 & unk & Coconut & NA & No lesions & - & - & - & - & - & - & - & + \\
\hline $17-150$ & 12 & $M$ & Rainbow & NA & No lesions & - & - & - & - & - & - & - & - \\
\hline $17-1689$ & 13 & $\mathrm{M}$ & Rainbow & NA & No lesions & - & - & - & - & - & - & - & - \\
\hline $17-988$ & 13 & unk & Rainbow & NA & No lesions & - & - & - & - & - & - & - & - \\
\hline $19-1244$ & 14.9 & M & Rainbow & NA & No lesions & - & - & - & - & - & - & - & - \\
\hline
\end{tabular}

Additional Files

890 Additional File 1: Experimental design. Lorikeet sampling by season and opportunistically

891 during cases of enteritis at the a) Columbus Zoo and Aquarium and the b) Denver Zoo. 
Additional File 2: Clostridium perfringens IHC protocol from California Animal Health \&

Additional File 3: Reagent preparation for measurement of Trypsin Inhibitor Levels in

nectar

Additional File 4: Microbial community diversity in healthy CZA birds by sex, species, and age. Healthy birds across all time points: Microbial diversity (Shannon Diversity Index and Observed Features) did not differ significantly by a,d) sex, b,e) species, or c,f) age (Kruskal Wallis: Shannon sex $p=0.77$, species $p=0.25$, age $p=0.89$; Observed Features sex $p=0.93$,

903 species $p=0.18$, age $p=0.08$ ).

Additional File 5: CZA Microbial community composition by sex, species, and age.

906 community composition (Unweighted UniFrac) and b) diversity (Shannon Diversity Index) by season (November 2018, February 2019, September 2019). There were significant shifts in

910 microbial composition by season (PERMANOVA $p=0.001$ ), and microbial diversity decreased

911 significantly between November 2018 and September 2019 (Kruskal-Wallis $* p<0.001$ ). (Also 912 see Fig. 1.) 
916 lorikeets that died or were euthanized due to enteritis (post-mortem). a) Microbial composition

917 (Unweighted UniFrac) was significantly altered (PERMANOVA $p=0.001$ ) and b) microbial

918 diversity (Shannon Diversity Index) was significantly decreased (Kruskal-Wallis * $p<0.005,{ }^{* *} p$

$919<0.0005, * * * p<0.00001)$ in lorikeets with enteritis or post-mortem lorikeets. (Also see Fig. 2.)

921 Additional File 8: Differentially abundant microbes by health status. Based on an ANCOM,

922 Clostridia were significantly increased in relative abundance in lorikeets with enteritis as

923 compared to healthy lorikeets. This analysis included 157 healthy samples and 45 enteritis

924 samples from a total of 67 birds. No post-mortem samples were included in this analysis.

925 Clostridia are in bold text.

926

927 Additional File 9: Differentially abundant microbes by health status in 25 birds with

928 matched healthy / enteritis samples. Based on an ANCOM, Clostridia were significantly

929 increased in relative abundance in lorikeets with enteritis as compared to healthy lorikeets. This

930 analysis included 29 healthy samples and 35 enteritis samples from a total of 25 birds. No post-

931 mortem samples were included in this analysis. Clostridia are in bold text.

933 Additional File 10: Denver Zoo (DZ) Lorikeet Demographics. The p-value for sex were based

934 on a $\chi^{2}$ test. A Kruskal-Wallis test was used to calculate the p-value for age.

935 The Freeman-Halton extension of the Fisher's Exact test was used to calculate a p-value for

936 species. 


\section{Additional File 11: Altered microbial composition and diversity in Denver Zoo lorikeets}

with enteritis. Microbial composition and diversity in healthy lorikeets and lorikeets that died or

Additional File 12: Susceptible CZA lorikeets have altered microbial composition that

945 predicts enteritis. Healthy lorikeets that never developed enteritis throughout the sampling

946 period were identified as "True Healthy" while healthy birds that developed enteritis at least

947 once during the sampling period were identified as "Susceptible." "Enteritis" represents birds

948 with enteritis that were sampled while they were clinically ill. No post-mortem samples are

949 included in this figure. a) Microbial diversity (Shannon, Kruskal-Wallis, $p<0.005$ ) was

950 increased in Susceptible and True Healthy birds as compared to birds with enteritis and b)

951 microbial composition (Unweighted UniFrac) was altered in Susceptible birds. Variables

952 associated with susceptibility or health were then predicted by a c) Random Forest (RF) or d)

953 LASSO model. The RF model has a sensitivity of 0.75 , a specificity of 0.571 , and an overall

954 accuracy of 0.557 . This model identifies the relative importance of each variable but not whether

955 the variable is associated with susceptibility or health. The LASSO model has a sensitivity of

9560.875 , a specificity of 0.571 , an overall accuracy of 0.733 , and generates an area under the curve

957 (AUC) of 0.72. This model (LASSO) identifies whether a variable is associated with

958 susceptibility or health but not the relative importance of the variable.

959

960

\section{References}


962 1. Ward MP, Ramer JC, Proudfoot J, Garner MM, Juan-Sallés C, Wu CC: Outbreak of

963 Salmonellosis in a Zoologic Collection of Lorikeets and Lories (Trichoglossus, Lorius, and Eos spp.). Avian Diseases 2003, 47(2):493-498.

2. Shima AL, Osborn KG: An Epornitic of Salmonella typhimurium in a Collection of Lories and Lorikeets. Journal of Zoo and Wildlife Medicine 1989, 20(3):373-376.

3. Mackie JT, Black D, Prior H: Enteritis associated with adeno-virus-like particles in captive lorikeets. Australian Veterinary Journal 2003, 81(5):293-295.

\section{Ferrell ST, Tell L: Clostridium tertium Infection in a Rainbow Lorikeet} (Trichoglossus haematodus haematodus) with Enteritis. Journal of Avian Medicine and Surgery 2001, 15(3):204-208.

5. Karunakaran N, Nagarajan K, Prathiba A, Soundararajan C, Bharathi SV: Incidence, Diagnosis and Treatment of Clostridial Enteritis in Lories and Lorikeets. Intas Polivet 2018, 19:385+.

6. Melander RJ, Zurawski DV, Melander C: Narrow-Spectrum Antibacterial Agents. Medchemcomm 2018, 9(1):12-21.

7. Sze MA, Schloss PD: Leveraging Existing 16S rRNA Gene Surveys To Identify Reproducible Biomarkers in Individuals with Colorectal Tumors. mBio 2018, 9(3):e00630-00618.

8. Bhatt AP, Redinbo MR, Bultman SJ: The role of the microbiome in cancer development and therapy. CA: a cancer journal for clinicians 2017, 67(4):326-344.

9. Wen C, Zheng Z, Shao T, Liu L, Xie Z, Le Chatelier E, He Z, Zhong W, Fan Y, Zhang L et al: Quantitative metagenomics reveals unique gut microbiome biomarkers in ankylosing spondylitis. Genome Biology 2017, 18(1):142.

10. Lemon KP, Armitage GC, Relman DA, Fischbach MA: Microbiota-Targeted Therapies: An Ecological Perspective. Science Translational Medicine 2012, 4(137):137rv135.

11. Young VB: The role of the microbiome in human health and disease: an introduction for clinicians. BMJ 2017, 356:j831.

12. Hacquard S, Garrido-Oter R, González A, Spaepen S, Ackermann G, Lebeis S, McHardy Alice C, Dangl Jeffrey L, Knight R, Ley R et al: Microbiota and Host Nutrition across Plant and Animal Kingdoms. Cell Host \& Microbe 2015, 17(5):603-616.

13. Round JL, Mazmanian SK: The gut microbiota shapes intestinal immune responses during health and disease. Nat Rev Immunol 2009, 9(5):313-323.

14. Kamada N, Chen GY, Inohara N, Núñez G: Control of Pathogens and Pathobionts by the Gut Microbiota. Nature immunology 2013, 14(7):685-690.

15. Pickard JM, Zeng MY, Caruso R, Núñez G: Gut microbiota: Role in pathogen colonization, immune responses, and inflammatory disease. Immunol Rev 2017, 279(1):70-89.

1001

1002

1003

1004

1005

16. Waite DW, Deines P, Taylor MW: Gut Microbiome of the Critically Endangered New Zealand Parrot, the Kakapo (Strigops habroptilus). PLOS ONE 2012, 7(4):e35803.

17. Xenoulis PG, Gray PL, Brightsmith D, Palculict B, Hoppes S, Steiner JM, Tizard I, Suchodolski JS: Molecular characterization of the cloacal microbiota of wild and captive parrots. Vet Microbiol 2010, 146:320-325.

1006

18. Ryu H, Grond K, Verheijen B, Elk M, Buehler DM, Santo Domingo JW: IIntestinal Microbiota and Species Diversity of Campylobacter and Helicobacter spp. in 
Migrating Shorebirds in Delaware Bay. Applied and Environmental Microbiology 2014, 80(6): 1838.

19. Hale VL, Tan CL, Niu K, Yang Y, Knight R, Zhang Q, Cui D, Amato KR: Diet Versus Phylogeny: a Comparison of Gut Microbiota in Captive Colobine Monkey Species. Microbial Ecology 2018, 75(2):515-527.

20. Bolnick DI, Snowberg LK, Hirsch PE, Lauber CL, Knight R, Caporaso JG, Svanbäck R: Individuals' diet diversity influences gut microbial diversity in two freshwater fish (threespine stickleback and Eurasian perch). Ecology Letters 2014, 17(8):979-987.

21. Escallón C, Belden LK, Moore IT: The Cloacal Microbiome Changes with the Breeding Season in a Wild Bird. Integrative Organismal Biology 2019, 1(1).

22. Kueneman JG, Woodhams DC, Harris R, Archer HM, Knight R, McKenzie VJ: Probiotic treatment restores protection against lethal fungal infection lost during amphibian captivity. Proceedings Biological sciences 2016, 283(1839):20161553.

23. Amato KR, Metcalf JL, Song SJ, Hale VL, Clayton J, Ackermann G, Humphrey G, Niu $\mathrm{K}$, Cui $\mathrm{D}$, Zhao $\mathrm{H}$ et al: Using the gut microbiota as a novel tool for examining colobine primate GI health. Global Ecology and Conservation 2016, 7:225-237.

24. Avena CV, Parfrey LW, Leff JW, Archer HM, Frick WF, Langwig KE, Kilpatrick AM, Powers KE, Foster JT, McKenzie VJ: Deconstructing the Bat Skin Microbiome: Influences of the Host and the Environment. Frontiers in Microbiology 2016, 7(1753).

25. Kohl KD, Weiss RB, Cox J, Dale C, Denise Dearing M: Gut microbes of mammalian herbivores facilitate intake of plant toxins. Ecology Letters 2014, 17(10):1238-1246.

26. Stumpf RM, Gomez A, Amato KR, Yeoman CJ, Polk JD, Wilson BA, Nelson KE, White BA, Leigh SR: Microbiomes, metagenomics, and primate conservation: New strategies, tools, and applications. Biological Conservation 2016, 199:56-66.

27. Clayton JB, Gomez A, Amato K, Knights D, Travis DA, Blekhman R, Knight R, Leigh $\mathrm{S}$, Stumpf R, Wolf $\mathrm{T}$ et al: The gut microbiome of nonhuman primates: Lessons in ecology and evolution. American Journal of Primatology 2018, 80(6):e22867.

28. West AG, Waite DW, Deines P, Bourne DG, Digby A, McKenzie VJ, Taylor MW: The microbiome in threatened species conservation. Biological Conservation 2019, 229:85-98.

29. Keyburn AL, Yan X-X, Bannam TL, Van Immerseel F, Rood JI, Moore RJ: Association between avian necrotic enteritis and Clostridium perfringens strains expressing NetB toxin. Veterinary research 2010, 41(2):21-21.

30. Shane $\mathrm{S}$ : The significance of campylobacter jejuni infection in poultry: A review Avian Pathology 1992, 21(2):189-213.

31. Pisani J, Speer B, Howerth EW, Clubb SL: Clostridial Infections in Psittacine Birds. Journal of Avian Medicine and Surgery 1998, 12(3):202-204.

32. O'Toole D, Mills K, Ellis R, Farr R, Davis M: Clostridial Enteritis in Red Lories (Eos Bounea). Journal of Veterinary Diagnostic Investigation 1993, 5(1):111-113.

33. Guimarães MB, Torres LN, Mesquita RG, Ampuero F, Cunha MPV, Ferreira TSP, Ferreira AJP, Catão-Dias JL, Moreno AM, Knöbl T: Clostridium perfringens Type A Enteritis in Blue and Yellow Macaw (Ara ararauna). Avian Diseases 2014, 58(4):650653, 654.

34. Asaoka Y, Yanai T, Hirayama H, Une Y, Saito E, Sakai H, Goryo M, Fukushi H, Masegi $\mathrm{T}$ : Fatal necrotic enteritis associated with Clostridium perfringens in wild crows (Corvus macrorhynchos). Avian Pathology 2004, 33(1):19-24. 
1053

1054

1055

1056

1057

1058

1059

1060

1061

1062

1063

1064

1065

1066

1067

1068

1069

1070

1071

1072

1073

1074

1075

1076

1077

1078

1079

1080

1081

1082

1083

1084

1085

1086

1087

1088

1089

1090

1091

1092

1093

1094

1095

1096

1097

35. Buffie CG, Bucci V, Stein RR, McKenney PT, Ling L, Gobourne A, No D, Liu H, Kinnebrew M, Viale A et al: Precision microbiome reconstitution restores bile acid mediated resistance to Clostridium difficile. Nature 2015, 517(7533):205-208.

36. Battaglioli EJ, Hale VL, Chen J, Jeraldo P, Ruiz-Mojica C, Schmidt BA, Rekdal VM, Till LM, Huq L, Smits SA et al: Clostridioides difficile uses amino acids associated with gut microbial dysbiosis in a subset of patients with diarrhea. Science Translational Medicine 2018, 10(464).

37. Ferreira RBR, Gill N, Willing BP, Antunes LCM, Russell SL, Croxen MA, Finlay BB: The intestinal microbiota plays a role in Salmonella-induced colitis independent of pathogen colonization. PloS one 2011, 6(5):e20338-e20338.

38. Gray KL: After 14 lorikeets die, zoo hopes illness gone. In: Columbus Dispatch. 2012.

39. Uzal FA, Vidal JE, McClane BA, Gurjar AA: Clostridium Perfringens Toxins Involved in Mammalian Veterinary Diseases. Open Toxinology J 2010, 2:24-42.

40. Liu K: Soybean Trypsin Inhibitor Assay: Further Improvement of the Standard Method Approved and Reapproved by American Oil Chemists' Society and American Association of Cereal Chemists International. Journal of the American Oil Chemists' Society 2019, 96(6):635-645.

41. Droual R, Farver TB, Bickford AA: Relationship of Sex, Age, and Concurrent Intestinal Disease to Necrotic Enteritis in Turkeys. Avian Diseases 1995, 39(3):599605.

42. Wobeser G, Rainnie DJ: EPIZOOTIC NECROTIC ENTERITIS IN WILD GEESE. Journal of Wildlife Diseases 1987, 23(3):376-385.

43. Silva ROS, Lobato FCF: Clostridium perfringens: A review of enteric diseases in dogs, cats and wild animals. Anaerobe 2015, 33:14-17.

44. Shivaprasad HL, Uzal F, Kokka R, Fisher DJ, McClane BA, Songer AG: Ulcerative Enteritis-Like Disease Associated with Clostridium perfringens Type A in Bobwhite Quail (Colinus virginianus). Avian Diseases 2008, 52(4):635-640.

45. McOrist S, Reece RL: Clostridial enteritis in free-living lorikeets (Trichoglossus spp.). Avian Pathology 1992, 21(3):503-507.

46. Rupiper DJ: Hemorrhagic Enteritis in a Group of Great-Billed Parrots (Tanygnathus megalorynchos). Journal of the Association of Avian Veterinarians 1993, 7(4):209-211.

47. Grau-Roma L, Navarro M, Blatter S, Wenker C, Kittl S, Uzal FA, Posthaus H: Clostridium perfringens-Associated Necrotic Enteritis-Like Disease in Coconut Lorikeets (Trichoglossus haematodus). Veterinary Pathology 2021, 58(2):423-427.

48. Ritchie BW, Harrison GJ, Harrison LR: Avian Medicine: Principles and Application. Lake Worth, FL: Wingers Publishing, Inc.; 1994.

49. Bildfell RJ, Eltzroth EK, Songer JG: Enteritis as a Cause of Mortality in the Western Bluebird (Sialia mexicana). Avian Diseases 2001, 45(3):760-763.

50. Góngora E, Elliott KH, Whyte L: Gut microbiome is affected by inter-sexual and inter-seasonal variation in diet for thick-billed murres (Uria lomvia). Scientific Reports 2021, 11(1):1200.

51. Drovetski SV, O’Mahoney MJV, Matterson KO, Schmidt BK, Graves GR: Distinct microbiotas of anatomical gut regions display idiosyncratic seasonal variation in an avian folivore. Animal Microbiome 2019, 1(1):2. 
52. Lewis WB, Moore FR, Wang S: Characterization of the gut microbiota of migratory passerines during stopover along the northern coast of the Gulf of Mexico. Journal of Avian Biology 2016, 47(5):659-668.

53. Le Roy CI, Woodward MJ, Ellis RJ, La Ragione RM, Claus SP: Antibiotic treatment triggers gut dysbiosis and modulates metabolism in a chicken model of gastrointestinal infection. BMC Veterinary Research 2019, 15(1):37.

54. Langdon A, Crook N, Dantas G: The effects of antibiotics on the microbiome throughout development and alternative approaches for therapeutic modulation. Genome medicine 2016, 8(1):39-39.

55. Dethlefsen L, Huse S, Sogin ML, Relman DA: The Pervasive Effects of an Antibiotic on the Human Gut Microbiota, as Revealed by Deep 16S rRNA Sequencing. PLOS Biology 2008, 6(11):e280.

56. Dethlefsen L, Relman DA: Incomplete recovery and individualized responses of the human distal gut microbiota to repeated antibiotic perturbation. Proc Natl Acad Sci 2011, 108 (1):4554-4561.

57. Modi SR, Collins JJ, Relman DA: Antibiotics and the gut microbiota. J Clin Investig 2014, 124(10):4212-4218.

58. Lu J, Hofacre C, Smith F, Lee MD: Effects of feed additives on the development on the ileal bacterial community of the broiler chicken. Animal 2008, 2(5):669-676.

59. Uzal FA, Navarro MA, Li J, Freedman JC, Shrestha A, McClane BA: Comparative pathogenesis of enteric clostridial infections in humans and animals. Anaerobe 2018, 53:11-20.

60. Cooper KK, Songer JG, Uzal FA: Diagnosing clostridial enteric disease in poultry. Journal of Veterinary Diagnostic Investigation 2013, 25(3):314-327.

61. Pattison M, McMullin P, Bradbury J, Alexander D (eds.): Poultry Diseases (Chapter 18, Clostridia), 6th edn: Saunders Ltd.; 2007.

62. Altman RB, Clubb SL, Dorrestein GM, Quesenberry K (eds.): Avian Medicine and Surgery: Saunders; 1997.

63. Pizarro M, Höfle U, Rodríguez-Bertos A, González-Huecas M, Castaño M: Ulcerative Enteritis (Quail Disease) in Lories. Avian Diseases 2005, 49(4):606-608.

64. Stanley D, Keyburn AL, Denman SE, Moore RJ: Changes in the caecal microflora of chickens following Clostridium perfringens challenge to induce necrotic enteritis. Veterinary Microbiology 2012, 159(1):155-162.

65. Lin Y, Xu S, Zeng D, Ni X, Zhou M, Zeng Y, Wang H, Zhou Y, Zhu H, Pan K et al: Disruption in the cecal microbiota of chickens challenged with Clostridium perfringens and other factors was alleviated by Bacillus licheniformis supplementation. PLOS ONE 2017, 12(8):e0182426.

66. Antonissen G, Eeckhaut V, Van Driessche K, Onrust L, Haesebrouck F, Ducatelle R, Moore RJ, Van Immerseel F: Microbial shifts associated with necrotic enteritis. Avian Pathology 2016, 45(3):308-312.

67. Fasina YO, Newman MM, Stough JM, Liles MR: Effect of Clostridium perfringens infection and antibiotic administration on microbiota in the small intestine of broiler chickens. Poultry Science 2016, 95(2):247-260.

68. Hadley TL: Disorders of the Psittacine Gastrointestinal Tract. Veterinary Clinics of North America: Exotic Animal Practice 2005, 8(2):329-349. 
69. Wilson GH, Ritchie BW, Greenacre CB, Fontenot D: Clostridium: Passenger or pathogen? In: Annual Conference of the Association of Avian Veterinarians: 1999. 251253.

70. Boujon P, Henzi M, Penseyres JH, Belloy L: Enterotoxaemia involving 及2-toxigenic Clostridium perfringens in a white stork (Ciconia ciconia). Veterinary Record 2005, 156(23):746-747.

71. Garmory HS, Chanter N, French NP, Bueschel D, Songer JG, Titball RW: Occurrence of Clostridium perfringens beta2-toxin amongst animals, determined using

72. Uzal FA, Songer JG, Prescott JF, Popoff MR (eds.): Clostridial Diseases of Animals: John Wiley \& Sons, Inc.; 2016.

73. Silva ROS, Junior FCF, Marques MVR, Junior CAO, Martins NRD, Lobato FCF: Genotyping and antimicrobial susceptibility of Clostridium perfringens isolated from Tinamidae, Cracidae and Ramphastidae species in Brazil. Ciência Rural 2014, 44(3):486-491.

74. Silva ROS, Ribeiro MG, Palhares MS, Borges AS, Maranhão RPA, Silva MX, Lucas TM, Olivo G, Lobato FCF: Detection of A/B toxin and isolation of Clostridium difficile and Clostridium perfringens from foals. Equine Veterinary Journal 2013, 45(6):671-675.

75. Silva ROS, Santos RLR, Pires PS, Pereira LC, Pereira ST, Duarte MC, de Assis RA, Lobato FCF: Detection of toxins A/B and isolation of Clostridium difficile and Clostridium perfringens from dogs in Minas Gerais, Brazil. Brazilian journal of microbiology : [publication of the Brazilian Society for Microbiology] 2013, 44(1):133137.

76. Schotte U, Truyen U, Neubauer H: Significance of $\beta 2$-Toxigenic Clostridium perfringens Infections in Animals and Their Predisposing Factors - A Review. Journal of Veterinary Medicine, Series B 2004, 51(10):423-426.

77. Crespo R, Fisher DJ, Shivaprasad HL, Fernández-Miyakawa ME, Uzal FA: Toxinotypes of Clostridium Perfringens Isolated from Sick and Healthy Avian Species. Journal of Veterinary Diagnostic Investigation 2007, 19(3):329-333.

78. Berto G, Agnoletti F, Drigo I, Tonon E, Vascellari M, Fracas V, Bano L: Clostridial coinfection episodes in commercial laying hens. Avian Pathology 2015, 44(3):200-203.

79. Forero AJ, Muñoz M, Camargo M, Soto-De León SC, Ríos-Chaparro DI, Birchenall C, Pinilla D, Pardo JM, Josa DF, Patarroyo MA et al: High frequency of toxigenic Clostridium difficile and Clostridium perfringens coinfection among diarrheic patients at health care facility-onset (HCFO) and community-onset (CO) centers in Bogotá, Colombia. Gut Pathogens 2019, 11(1):27.

80. Uzal FA, Diab SS, Blanchard P, Moore J, Anthenill L, Shahriar F, Garcia JP, Songer JG: Clostridium perfringens type $\mathbf{C}$ and Clostridium difficile co-infection in foals. Veterinary Microbiology 2012, 156(3):395-402.

81. Diniz AN, Silva ROS, Oliveira Junior CA, Pierezan F, Lobato FCF: Clostridium perfringens type $A$ netF and netE positive and Clostridium difficile co-infection in two adult dogs. Anaerobe 2016, 38:94-96. Liu CM, Hayer M, McHugh TA et al: Phylogenetic organization of bacterial activity. The ISME Journal 2016, 10(9):2336-2340. 
83. Hermans PG, Morgan KL: Prevalence and associated risk factors of necrotic enteritis on broiler farms in the United Kingdom; a cross-sectional survey. Avian Pathology 2007, 36(1):43-51.

84. Kaldhusdal M, Skjerve E: Association between cereal contents in the diet and incidence of necrotic enteritis in broiler chickens in Norway. Preventive Veterinary Medicine 1996, 28(1):1-16.

85. Fancher CA, Zhang L, Kiess AS, Adhikari PA, Dinh TTN, Sukumaran AT: Avian Pathogenic Escherichia coli and Clostridium perfringens: Challenges in No Antibiotics Ever Broiler Production and Potential Solutions. Microorganisms 2020, 8(10): 1533.

86. Gerlach H: Bacteria. In: Avian Medicine: Principles and Applications. Edited by Ritchie B, Harrison G, Harrison L. Lake Worth, FL: Wingers Publishing, Inc.; 1994: 949-983.

87. Gibert M, Jolivet-Renaud C, Popoff MR: Beta2 toxin, a novel toxin produced by Clostridium perfringens. Gene 1997, 203(1):65-73.

88. Palliyeguru MWCD, Rose SP, Mackenzie AM: Effect of trypsin inhibitor activity in soya bean on growth performance, protein digestibility and incidence of sub-clinical necrotic enteritis in broiler chicken flocks. British Poultry Science 2011, 52(3):359367.

89. Bradbury JH, Hammer B, Tue N, Anders M, Millar JS: Protein quantity and quality and trypsin inhibitor content of sweet potato cultivars from the highlands of Papua New Guinea. Journal of Agricultural and Food Chemistry 1985, 33(2):281-285.

90. Zhang Z, Corke H: Trypsin inhibitor activity in vegetative tissue of sweet potato plants and its response to heat treatment. Journal of the Science of Food and Agriculture 2001, 81(14):1358-1363.

91. Senanayake SA, Ranaweera KKDS, Gunaratne A, Bamunuarachchi A: Comparative analysis of nutritional quality of five different cultivars of sweet potatoes (Ipomea batatas (L) Lam) in Sri Lanka. Food Science \& Nutrition 2013, 1(4):284-291.

92. Lacey JA, Stanley D, Keyburn AL, Ford M, Chen H, Johanesen P, Lyras D, Moore RJ: Clostridium perfringens-mediated necrotic enteritis is not influenced by the preexisting microbiota but is promoted by large changes in the post-challenge microbiota. Veterinary Microbiology 2018, 227:119-126.

93. Kiu R, Hall LJ: An update on the human and animal enteric pathogen Clostridium perfringens. Emerg Microbes Infect 2018, 7(1):141-141.

94. Sorg JA, Sonenshein AL: Bile salts and glycine as cogerminants for Clostridium difficile spores. Journal of bacteriology 2008, 190(7):2505-2512.

95. Austin MC, Hallstrand TS, Hoogestraat DR, Balmforth G, Stephens K, Butler-Wu S, Yeung CCS: Rhodococcus fascians infection after haematopoietic cell transplantation: not just a plant pathogen? JMM Case Rep 2016, 3(2):e005025e005025.

96. Glünder G, Siegmann O: Iccurrence of aeromonas hydrophila in wild birds. Avian Pathology 1989, 18(4):685-695.

97. França M, Walker RL, Kokka R, Shivaprasad HL: Aeromonas Species Associated with Necrotizing Enteritis and Septicemia in an Adult Male Ostrich (Struthio camelus). Avian Diseases 2009, 53(2):310-316.

98. Brook I, Walker RI: Pathogenicity of Clostridium species with other bacteria in mixed infections. Journal of Infection 1986, 13(3):245-253. 
99. Keyburn AL, Sheedy SA, Ford ME, Williamson MM, Awad MM, Rood JI, Moore RJ: Alpha-toxin of Clostridium perfringens is not an essential virulence factor in necrotic enteritis in chickens. Infection and immunity 2006, 74(11):6496-6500.

100. Brzęk P, Ciminari ME, Kohl KD, Lessner K, Karasov WH, Caviedes-Vidal E: Effect of age and diet composition on activity of pancreatic enzymes in birds. J Comp Physiol B 2013, 183(5):685-697.

101. Bolyen E, Rideout JR, Dillon MR, Bokulich NA, Abnet CC, Al-Ghalith GA, Alexander $\mathrm{H}$, Alm EJ, Arumugam M, Asnicar F et al: Reproducible, interactive, scalable and extensible microbiome data science using QIIME 2. Nature Biotechnology 2019, 37(8):852-857.

102. Callahan BJ, McMurdie PJ, Rosen MJ, Han AW, Johnson AJA, Holmes SP: DADA2: High-resolution sample inference from Illumina amplicon data. Nature methods 2016, 13(7):581-583.

103. AACC: AACC Method 22-40: Measurement of trypsin inhibitor activity of soy products - spectrophotometric method, 11th edn. St. Paul, MN: AACC International; 1999.

104. Preacher KJ: Calculation for the chi-square test: An interactive calculation tool for chi-square tests of goodness of fit and independence In.; 2001.

105. Mandal S, Van Treuren W, White RA, Eggesbø M, Knight R, Peddada SD: Analysis of composition of microbiomes: a novel method for studying microbial composition. Microb Ecol Health Dis 2015, 26:27663-27663.

106. Breiman L: Random Forests. Machine Learning 2001, 45(1):5-32.

107. Tibshirani R: Regression Shrinkage and Selection via the Lasso. Journal of the Royal Statistical Society Series B (Methodological) 1996, 58(1):267-288.

108. Efron B, Hastie T, Johnstone I, Tibshirani R: Least angle regression. The Annals of Statistics 2004, 32(2):407-499, 493. 